\title{
SATISFIABILITY AND MODEL CHECKING FOR THE LOGIC OF SUB-INTERVALS UNDER THE HOMOGENEITY ASSUMPTION*
}

\author{
LAURA BOZZELLI $^{a}$, ALBERTO MOLINARI $^{b}$, ANGELO MONTANARI $^{b}$, ADRIANO PERON $^{a}$, \\ AND PIETRO SALA ${ }^{c}$ \\ ${ }^{a}$ University of Napoli "Federico II", Napoli, Italy \\ e-mail address: Ir.bozzelli@gmail.com, adrperon@unina.it \\ ${ }^{b}$ University of Udine, Udine, Italy \\ e-mail address: molinari.alberto@gmail.com, angelo.montanari@uniud.it \\ ${ }^{c}$ University of Verona, Verona, Italy \\ e-mail address: pietro.sala@univr.it
}

\begin{abstract}
The expressive power of interval temporal logics (ITLs) makes them one of the most natural choices in a number of application domains, ranging from the specification and verification of complex reactive systems to automated planning. However, for a long time, because of their high computational complexity, they were considered not suitable for practical purposes. The recent discovery of several computationally well-behaved ITLs has finally changed the scenario.

In this paper, we investigate the finite satisfiability and model checking problems for the ITL D, that has a single modality for the sub-interval relation, under the homogeneity assumption (that constrains a proposition letter to hold over an interval if and only if it holds over all its points). We first prove that the satisfiability problem for D, over finite linear orders, is PSPACE-complete, and then we show that the same holds for its model checking problem, over finite Kripke structures. In such a way, we enrich the set of tractable interval temporal logics with a new meaningful representative.
\end{abstract}

\section{INTRODUCTION}

For a long time, interval temporal logics (ITLs) were considered an attractive, but impractical, alternative to standard point-based ones. On the one hand, as stated by Kamp and Reyle [KR93], "truth, as it pertains to language in the way we use it, relates sentences not to instants but to temporal intervals", and thus ITLs are a natural choice as a specification/representation language; on the other hand, the high undecidability of the satisfiability problem for the most well-known ITLs, such as Halpern and Shoham's modal logic of time intervals (HS for short) [HS91] and Venema's CDT [Ven91], prevented an extensive use of them (in fact, some quite restricted variants of them have been applied in formal verification and artificial intelligence over the years).

Key words and phrases: Interval Temporal Logics, Expressiveness, Model Checking.

* This paper is an extended and revised version of $\left[\mathrm{BMM}^{+} 17\right]$.

넴

LOGICAL METHODS IN COMPUTER SCIENCE
DOI:10.46298/LMCS-18(1:24)2022 (c) L. Bozzelli, A. Molinari, A. Montanari, A. Peron, and P. Sala

(C) Creative Commons 
Table 1: Allen's relations and the corresponding HS modalities.

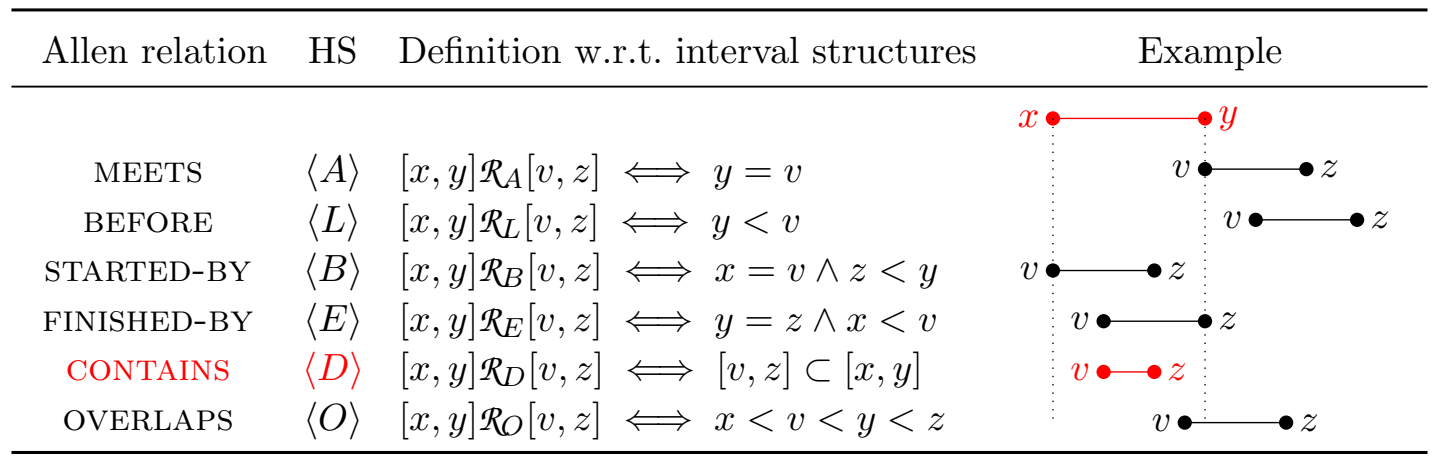

The present work fits into the context of the logic HS, which features one modality for each of the thirteen Allen's relations, apart from equality. In Table 1, we depict six Allen's relations, each one corresponding to a different ordering relation between a pair of intervals, together with the corresponding HS (existential) modalities. The other seven relations are the inverses of the six depicted ones, plus the equality relation.

The recent discovery of a significant number of expressive and computationally wellbehaved fragments of full HS changed the landscape of research in ITLs ( [DGMS11, Mon16]. Meaningful examples are the logic A $\bar{A}$ of temporal neighborhood [BGMS09] (the HS fragment featuring modalities for the meets relation and its inverse met by) and the logic $\mathrm{D}$ of (temporal) sub-intervals [BGMS10] (the HS fragment featuring only one modality for the contains relation). In this paper, we focus on the latter.

To begin with, we observe that $\mathrm{D}$ is a (proper) fragment of the logic BE with modalities for the Allen's relations started-by and finished-by (see Table 1) since any sub-interval is just an initial sub-interval of an ending one, or, equivalently, an ending sub-interval of an initial one. From a computational point of view, D is a real character. Indeed, it has been shown that its satisfiability problem is PSPACE-complete over the class of dense linear orders [BGMS10, Sha04] (the same problem is undecidable for BE [Lod00]), while it becomes undecidable when the logic is interpreted over the classes of finite and discrete linear orders [MM14]. The situation is still unknown over the class of all linear orders. As for its expressiveness, unlike $\mathrm{A} \overline{\mathrm{A}}$ - which is expressively complete with respect to the two-variable fragment of first-order logic for binary relational structures over various linearly-ordered domains [BGMS09, Ott01] - three variables are needed to encode D in first-order logic (the two-variable property is a sufficient condition for decidability, but it is not a necessary one).

In this paper, we show that the decidability of the satisfiability problem for $\mathrm{D}$ over the class of finite linear orders can be recovered by assuming homogeneity (the homogeneity assumption constrains a proposition letter to hold over an interval if and only if it holds over all its constituent points). First, by exploiting a suitable contraction method, we prove that, under the homogeneity assumption, the problem belongs to PSPACE. Then, we show that the proposed algorithm for satisfiability checking can be transformed into a PSPACE model-checking procedure for D formulas over finite Kripke structures (under homogeneity). Finally, PSPACE-hardness of both satisfiability and model checking is proved via a reduction from a domino-tiling problem for grid with rows of linear length.

The result of this paper about PSPACE-completeness of the model checking problem for $\mathrm{D}$ is particularly interesting when compared to the model checking problem for BE, which 
includes D as a proper fragment and, at first sight, may seem to be quite close to D. The exact complexity of the model checking problem for BE, over finite Kripke structures, under the homogeneity assumption, is still unknown and it is a difficult open question whether it can be solved elementarily. At the present time, only a nonelementary model checking procedure is known for it $\left[\mathrm{MMM}^{+} 16\right]$, and the closest proved lower bound is EXPSPACE $\left[\mathrm{BMM}^{+} 19 \mathrm{~b}\right]$. The complete picture for the complexity of the model checking (MC for short) problem for HS and its fragments (under homogeneity) is reported in Figure 1. Being D the most significant fragment of $\mathrm{BE}$, the results given in this paper allow us to gain a better insight into such an open question. The exact complexity of the satisfiability problem for BE over finite linear orders (under homogeneity) is an open issue as well: the same upper/lower bounds can be shown to hold by reductions to/from the MC problem.

Related work. Temporal logics of sub-intervals have been already investigated in the literature. In particular, they come into play in the study of temporal prepositions in natural language [Pra05]. The connections between the temporal logic of (strict) sub-intervals and the logic of Minkowski space-time have been explored by Shapirovsky and Shehtman [SS05], while the temporal logic of reflexive sub-intervals has been studied for the first time by van Benthem, who proved that, when interpreted over dense linear orderings, it is equivalent to the standard modal logic S4 [vB91]. Satisfiability for the temporal logic of reflexive subintervals over the class of finite linear orders is decidable and PSPACE-complete [MPS10].

The satisfiability problem for HS has been extensively studied in the literature. The problem in its full generality, that is, without the homogeneity assumption, has been proved to be highly undecidable over all relevant (classes of) linear orders [HS91]. The same holds for most of its fragments $\left[\mathrm{BDG}^{+} 14\right.$, Lod00, MM14].

The MC problem for HS and its fragments over finite Kripke structures (under the homogeneity assumption) has been systematically explored in a series of papers [LM13, LM14, $\mathrm{LM}_{16}, \mathrm{MMM}^{+} 16, \mathrm{MMP}$ 18, $\left.\mathrm{BMM}^{+} 18, \mathrm{BMM}^{+} 19 \mathrm{~b}\right]$. In Figure 1, we add the contribution of this paper to the general picture of known complexity results making it clear that it enriches the set of "tractable" fragments with a meaningful new member. An expressive comparison of the MC problem for HS and point-based temporal logics LTL, CTL, and CTL* can be found in $\left[\mathrm{BMM}^{+} 19 \mathrm{a}\right]$.

Organization of the paper. In Section 2, we provide some background knowledge. In particular, we introduce the logic D, the notion of interval model, and an extremely useful spatial representation of interval models called compass structure. In Section 3, we prove PSPACE membership of the satisfiability problem for D over finite linear orders (under homogeneity) making use of a contraction technique applied to compass structures, which relies on a suitable equivalence relation of finite index. In Section 4 , we show that the MC problem for D (under homogeneity), over finite Kripke structures, is in PSPACE as well. The proposed MC algorithm is basically a satisfiability procedure driven by the computation traces of the system model. In Section 5, we show PSPACE-hardness of both problems via a polynomial-time reduction from a domino-tiling problem for grids with rows of linear length. Conclusions summarize the work done and outline future research directions.

\section{The Logic D of the SUb-INTERVAl RElation}

To begin with, we introduce some preliminary notions. Let $\mathbb{S}=\langle S,<\rangle$ be a linear order over a nonempty set $S \neq \emptyset$, and $\leq$ be the reflexive closure of $<$. Given two elements $x, y \in S$, 


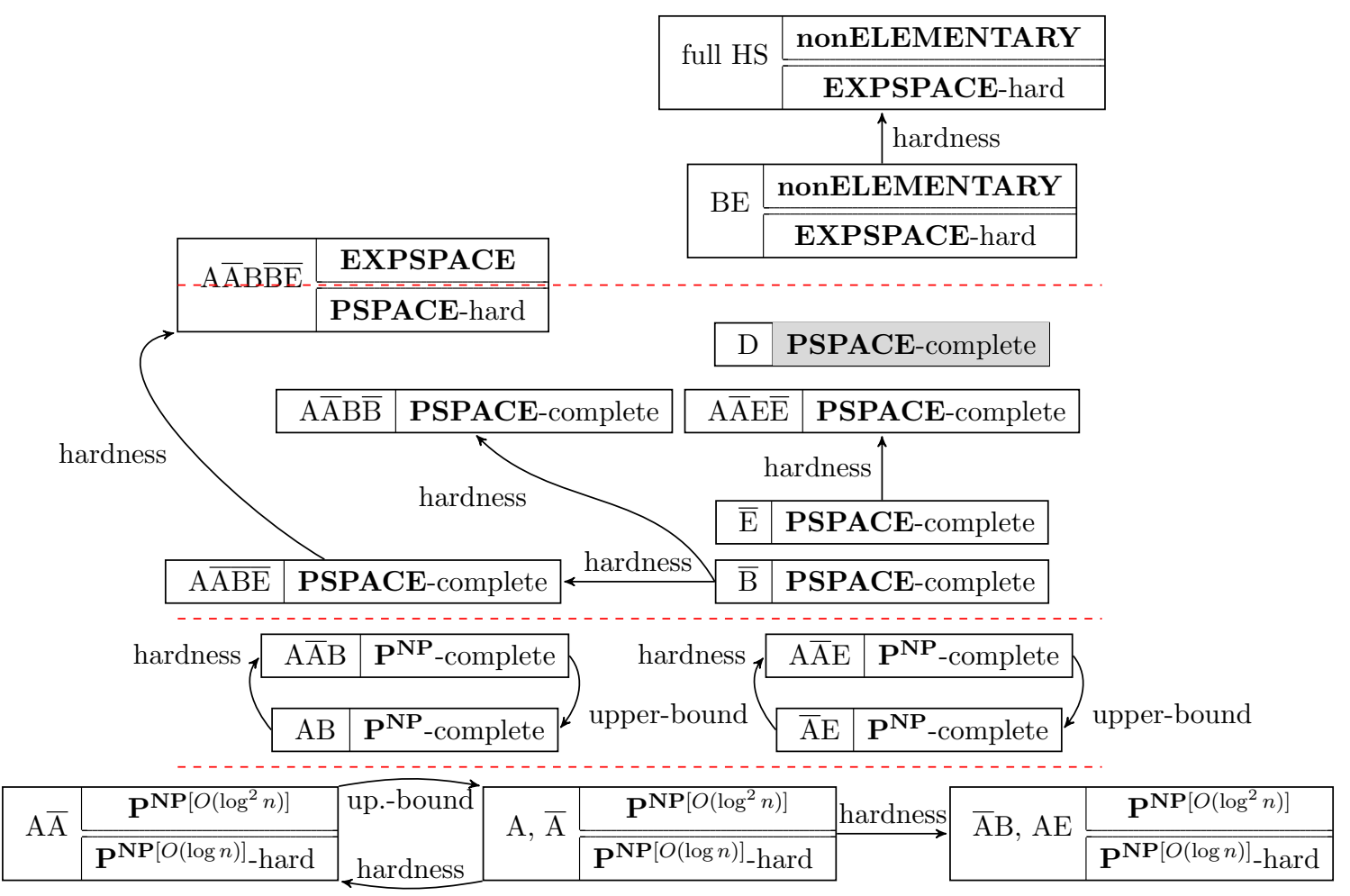

Figure 1: Complexity of the MC problem for HS and its fragments (under homogeneity).

with $x \leq y$, we denote by $[x, y]$ the (closed) interval over $S$ consisting of the set of elements $z \in S$ such that $x \leq z$ and $z \leq y$. For $z \in S$, we write $z \in[x, y]$ to mean that $z$ is an element of the interval $[x, y]$. We denote the set of all intervals over $\mathbb{S}$ by $\mathbb{I}(\mathbb{S})$. We consider two possible sub-interval relations:

(1) the proper sub-interval relation (denoted as $\sqsubset$ ), defined by $[x, y] \sqsubset\left[x^{\prime}, y^{\prime}\right]$ if $x^{\prime} \leq x$, $y \leq y^{\prime}$, and $[x, y] \neq\left[x^{\prime}, y^{\prime}\right]$ (corresponding to the proper subset relation over intervals),

(2) and the strict sub-interval relation (denoted as $\sqsubset$ ), defined by $[x, y] \leftarrow\left[x^{\prime}, y^{\prime}\right]$ if and only if $x^{\prime}<x$ and $y<y^{\prime}$.

The two modal logics $\mathrm{D}_{\sqsubset}$ and $\mathrm{D}_{\sqsubset}$ feature the same language, consisting of a finite set $\mathscr{A} \mathscr{P}$ of proposition letters/variables, the logical connectives $\neg$ and $\vee$, and the modal operator $\langle D\rangle$. Formally, formulae are defined by the grammar:

$$
\varphi::=p|\neg \varphi| \varphi \vee \varphi \mid\langle D\rangle \varphi,
$$

with $p \in \mathcal{A P}$. The other connectives, as well as the logical constants $\top$ (true) and $\perp$ (false), are defined as usual; moreover, the dual universal modality $[D] \varphi$ is defined as $\neg\langle D\rangle \neg \varphi$. The length of a formula $\varphi$, denoted as $|\varphi|$, is the number of sub-formulas of $\varphi$.

The semantics of $D_{\sqsubset}$ and $D_{\sqsubset}$ only differ in the interpretation of the $\langle D\rangle$ modality. For the sake of brevity, we use $\circ \in\{\sqsubset, \sqsubset\}$ as a shorthand for any of the two sub-interval relations. The semantics of a sub-interval logic $\mathrm{D}_{\circ}$ is defined in terms of interval models $\mathbf{M}=\langle\mathbb{I}(\mathbb{S}), \mathcal{V}\rangle$, where $\mathcal{V}: \mathcal{A P} \mapsto 2^{\mathbb{I}(\mathbb{S})}$ is a valuation function assigning to every proposition letter $p$ the set of intervals $\mathcal{V}(p)$ over which it holds. Given an interval model $\mathbf{M}=\langle\mathbb{I}(\mathbb{S}), \mathcal{V}\rangle$, 
an interval $[x, y] \in \mathbb{I}(\mathbb{S})$, and a formula $\psi$, the satisfaction relation $\mathbf{M},[x, y]=\psi$, stating that $\psi$ holds over the interval $[x, y]$ of $\mathbf{M}$, is inductively defined as follows:

- for every proposition letter $p \in \mathcal{A P}, \mathbf{M},[x, y] \models p$ if $[x, y] \in \mathcal{V}(p)$;

- $\mathbf{M},[x, y] \mid=\neg \psi$ if $\mathbf{M},[x, y] \not \models \psi$, that is, it is not true that $\mathbf{M},[x, y] \models \psi$;

- $\mathbf{M},[x, y]=\psi_{1} \vee \psi_{2}$ if either $\mathbf{M},[x, y] \models \psi_{1}$ or $\mathbf{M},[x, y] \models \psi_{2}$;

- $\mathbf{M},[x, y] \models\langle D\rangle \psi$ if there exists an interval $\left[x^{\prime}, y^{\prime}\right] \in \mathbb{I}(\mathbb{S})$ such that $\left[x^{\prime}, y^{\prime}\right] \circ[x, y]$ and $\mathbf{M},\left[x^{\prime}, y^{\prime}\right] \mid \psi \psi$

A $\mathrm{D}_{\circ}$-formula is $\mathrm{D}_{\circ}$-satisfiable if it holds over some interval of an interval model, and it is $\mathrm{D}_{\circ}$-valid if it holds over every interval of every interval model.

In this paper, we restrict our attention to the finite satisfiability problem, that is, satisfiability over the class of finite linear orders. The problem has been shown to be undecidable for $\mathrm{D}_{\sqsubset}$ and $\mathrm{D}_{\sqsubset}$ [MM14]. In the following, we show that decidability for $\mathrm{D}_{\sqsubset}$ and $\mathrm{D}_{\mp}$ can be recovered by restricting to the class of homogeneous interval models. We fully work out the case of $\mathrm{D}_{\sqsubset}$ (for the sake of simplicity, hereafter we will write $\mathrm{D}$ for $\mathrm{D}_{\sqsubset}$ ), and then we briefly explain how to adapt the proofs to $\mathrm{D}_{\sqsubset}$.

Definition 2.1. A model $\mathbf{M}=\langle\mathbb{I}(\mathbb{S}), \mathcal{V}\rangle$ is homogeneous if for every interval $[x, y] \in \mathbb{I}(\mathbb{S})$ and every $p \in \mathcal{A P}$, it holds that $[x, y] \in \mathcal{V}(p)$ if and only if $\left[x^{\prime}, x^{\prime}\right] \in \mathcal{V}(p)$ for every $x^{\prime} \in[x, y]$.

In the following, we will refer to $\mathrm{D}$ interpreted over homogeneous models as $\left.\mathrm{D}\right|_{\mathcal{H} \text { om }}$. Moreover, we refer to $\mathrm{D}_{\sqsubset}$ interpreted over homogeneous models as $\left.\mathrm{D}\right|_{\mathcal{H} \text { om, } 匚 \text {. }}$.

2.1. Closure, atoms, and temporal requests. We now introduce some basic definitions and notation which will be extensively used in the following. Given a D-formula (resp., $\mathrm{D}_{\leftarrow-\text {-formula) }} \varphi$, we define the closure of $\varphi$, denoted by $\mathrm{CL}(\varphi)$, as the set of all sub-formulas $\psi$ of $\varphi$ and of their negations $\neg \psi$ (we identify $\neg \neg \psi$ with $\psi$ and $\neg\langle D\rangle \psi$ with $[D] \neg \psi$ ).

Definition 2.2. A $\varphi$-atom $A$ is a subset of $\mathrm{CL}(\varphi)$ such that:

- for every $\psi \in \mathrm{CL}(\varphi), \psi \in A$ if and only if $\neg \psi \notin A$, and

- for every $\psi_{1} \vee \psi_{2} \in \mathrm{CL}(\varphi), \psi_{1} \vee \psi_{2} \in A$ if and only if either $\psi_{1} \in A$ or $\psi_{2} \in A$.

Intuitively, a $\varphi$-atom describes a maximal set of sub-formulas of $\varphi$ which can hold at an interval of an interval model. In particular, the idea underlying atoms is to enforce a "local" (or Boolean) form of consistency among the formulas it contains, that is, a $\varphi$-atom $A$ is a maximal, locally consistent subset of $\operatorname{CL}(\varphi)$. As an example, $\neg\left(\psi_{1} \vee \psi_{2}\right) \in A$ iff $\neg \psi_{1} \in A$ and $\neg \psi_{2} \in A$. Note, however, that the definition does not set any constraint on sub-formulas of $\varphi$ of the form $\langle D\rangle \psi$, hence the word "local". We denote the set of all $\varphi$-atoms by $\mathcal{A}_{\varphi}$. Its cardinality is clearly bounded by $2^{|\varphi|}$ (by the first item of Definition 2.2). Atoms are connected by the following binary relation $D_{\varphi}$ which, intuitively, represents the "symbolic" counterpart of the inverse $\sqsupset$ of relation $\sqsubset$ between pairs of intervals, if $\varphi$ is a D-formula, and the inverse $\sqsupset$ of relation $\sqsubset$, otherwise.

Definition 2.3. Let $D_{\varphi}$ be the binary relation over $\mathcal{A}_{\varphi}$ defined as follows: for each pair of atoms $A, A^{\prime} \in \mathcal{A}_{\varphi}, A D_{\varphi} A^{\prime}$ holds if for all formulas $[D] \psi \in A$, both $\psi \in A^{\prime}$ and $[D] \psi \in A^{\prime}$.

Note that, by the semantics of $\mathrm{D}$ (resp., $\mathrm{D}_{\leftarrow}$ ), if $A$ and $A^{\prime}$ are the sets of formulas in $\operatorname{CL}(\varphi)$ that hold at intervals $[x, y]$ and $\left[x^{\prime}, y^{\prime}\right]$, respectively, of an interval model such that $[x, y] \sqsupset\left[x^{\prime}, y^{\prime}\right]$ (resp., $[x, y] \sqsupset\left[x^{\prime}, y^{\prime}\right]$ ), then $A D_{\varphi} A^{\prime}$.

Let $A$ be a $\varphi$-atom. We denote by $\mathcal{R} e q_{D}(A)$ the set $\{\psi \in \operatorname{CL}(\varphi):\langle D\rangle \psi \in A\}$ of "temporal requests" of $A$. In particular, if $\langle D\rangle \psi \in \mathrm{CL}(\varphi)$ and $\psi \notin \mathcal{R} e q_{D}(A)$, then $[D] \neg \psi \in A$ 


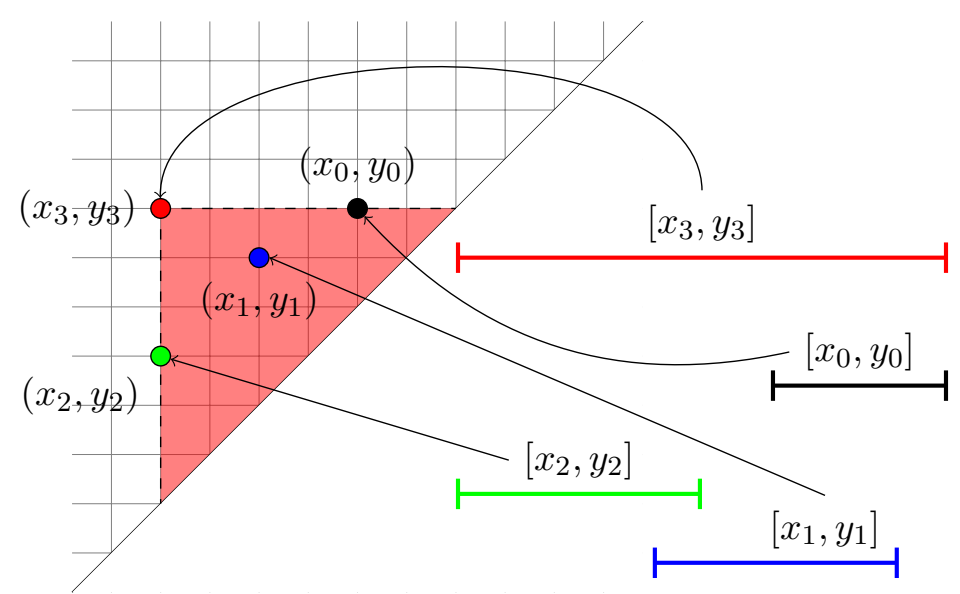

Figure 2: Correspondence between intervals and points of the compass structure.

(by definition of $\varphi$-atom). Moreover, we denote by $\mathrm{REQ}_{\varphi}$ the set of all arguments of $\langle D\rangle$ formulas in $\mathrm{CL}(\varphi)$, namely, $\mathrm{REQ}_{\varphi}=\{\psi:\langle D\rangle \psi \in \mathrm{CL}(\varphi)\}$. Finally, we denote by $\mathcal{O b s}_{D}(A)$ the set $\left\{\psi \in A: \psi \in \mathrm{REQ}_{\varphi}\right\}$ of "observables" of $A$.

The next proposition, stating that, once proposition letters and temporal requests of $A$ have been fixed, $A$ gets unambiguously determined, can be easily proved by induction.

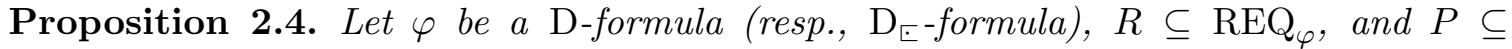
$\operatorname{CL}(\varphi) \cap \mathcal{A P}$. Then, there is a unique $\varphi$-atom $A$ that satisfies $\mathcal{R} e q_{D}(A)=R$ and $A \cap \mathcal{A P}=P$.

In the rest of the paper, we will exploit the following characterization of the relation $D_{\varphi}$.

Proposition 2.5. Let $A$ and $A^{\prime}$ be two $\varphi$-atoms. Then, $A D_{\varphi} A^{\prime}$ if and only if $\mathcal{R} e q_{D}\left(A^{\prime}\right) \subseteq$ $\mathcal{R} e q_{D}(A)$ and $\mathcal{O} b s_{D}\left(A^{\prime}\right) \subseteq \mathcal{R} e q_{D}(A)$.

Proof. We have that $A D_{\varphi} A^{\prime}$ if and only if for all $[D] \psi \in \mathrm{CL}(\varphi),[D] \psi \in A$ entails that $[D] \psi \in A^{\prime}$ and $\psi \in A^{\prime}$ if and only if (by definition of atom and the identification of $\neg\langle D\rangle \psi$ with $[D] \neg \psi$ ) for all $\langle D\rangle \psi \in \mathrm{CL}(\varphi)$, (i) $\langle D\rangle \psi \in A^{\prime}$ entails that $\langle D\rangle \psi \in A$, and (ii) $\psi \in A^{\prime}$ entails that $\langle D\rangle \psi \in A$ if and only if $\mathcal{R e q}_{D}\left(A^{\prime}\right) \subseteq \mathcal{R} e q_{D}(A)$ and $\mathcal{O} b s_{D}\left(A^{\prime}\right) \subseteq \mathcal{R} e q_{D}(A)$.

2.2. A spatial representation of interval models. We now provide a natural interpretation of $\mathrm{D}$ (resp., $\mathrm{D}_{\sqsubset}$ ) over grid-like structures, called compass structures, by exploiting the existence of a natural bijection between intervals $[x, y]$ and points $(x, y)$, with $x \leq y$, of an $S \times S$ grid, where $\mathbb{S}=\langle S,<\rangle$ is a finite linear order. Such an interpretation was originally proposed by Venema in [Ven90], and it can also be given for HS and all its (other) fragments.

As an example, Figure 2 shows four intervals $\left[x_{0}, y_{0}\right], \ldots,\left[x_{3}, y_{3}\right]$, respectively represented by the points in the grid $\left(x_{0}, y_{0}\right), \ldots,\left(x_{3}, y_{3}\right)$, such that: $(i)\left[x_{0}, y_{0}\right],\left[x_{1}, y_{1}\right],\left[x_{2}, y_{2}\right] \sqsubset\left[x_{3}, y_{3}\right]$, (ii) $\left[x_{1}, y_{1}\right] \sqsubset\left[x_{3}, y_{3}\right]$, and $($ iii $)\left[x_{0}, y_{0}\right],\left[x_{2}, y_{2}\right] \not \subset\left[x_{3}, y_{3}\right]$. The red region highlighted in Figure 2 contains all and only those points $(x, y)$ such that $[x, y] \sqsubset\left[x_{3}, y_{3}\right]$. Allen interval relation contains can thus be represented as a spatial relation between pairs of points. In the following, we make use of $\sqsubset$ and $\sqsubset$ also for relating points: given two points $(x, y),\left(x^{\prime}, y^{\prime}\right)$ of the grid, $\left(x^{\prime}, y^{\prime}\right) \sqsubset(x, y)$ iff $\left[x^{\prime}, y^{\prime}\right] \sqsubset[x, y]$; similarly, $\left(x^{\prime}, y^{\prime}\right) \sqsubset(x, y)$ iff $\left[x^{\prime}, y^{\prime}\right] \sqsubset[x, y]$.

Compass structures, that will be repeatedly exploited to solve the satisfiability and model-checking problems for $\left.\mathrm{D}\right|_{\mathcal{H} o m}$ and $\left.\mathrm{D}\right|_{\mathcal{H} o m, \Sigma}$, can be formally defined as follows. 
Definition 2.6. Given a linear order $\mathbb{S}=\langle S,<\rangle$ and a $\mathrm{D}$-formula (resp., $\mathrm{D}_{\leftarrow}$-formula) $\varphi$, a compass $\varphi$-structure is a pair $\mathcal{G}=\left(\mathbb{P}_{\mathbb{S}}, \mathcal{L}\right)$, where $\mathbb{P}_{\mathbb{S}}$ is the set of points of the form $(x, y)$, with $x, y \in S$ and $x \leq y$, and $\mathcal{L}$ is a function that maps any point $(x, y) \in \mathbb{P}_{\mathbb{S}}$ to a $\varphi$-atom $\mathcal{L}(x, y)$ in such a way that for every pair of points $(x, y),\left(x^{\prime}, y^{\prime}\right) \in \mathbb{P}_{\mathbb{S}}$, if $\left(x^{\prime}, y^{\prime}\right) \sqsubset(x, y)$ (resp., $\left.\left(x^{\prime}, y^{\prime}\right) \sqsubset(x, y)\right)$, then $\mathcal{L}(x, y) D_{\varphi} \mathcal{L}\left(x^{\prime}, y^{\prime}\right)$ (temporal consistency).

A weak compass $\varphi$-structure is a $\varphi$-compass structure where the temporal consistency requirement has been relaxed. A (weak) compass $\varphi$-structure $\mathcal{G}=\left(\mathbb{P}_{\mathbb{S}}, \mathcal{L}\right)$ induces the interval model $\mathbf{M}(\mathcal{G})$ over $\mathbb{I}(\mathbb{S})$ whose valuation function $\mathcal{V}$ is defined as follows: for each interval $[x, y], \mathcal{V}^{-1}([x, y])=\mathcal{L}(x, y) \cap \mathcal{A P}$.

By exploiting Proposition 2.5 and temporal consistency, we can prove the following lemma, that states an important property of compass structures.

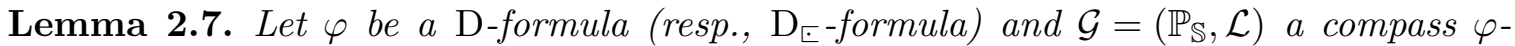
structure. For all pairs of points $\left(x^{\prime}, y^{\prime}\right),(x, y) \in \mathbb{P}_{\mathbb{S}}$, if $\left(x^{\prime}, y^{\prime}\right) \sqsubset(x, y)$ (resp., $\left.\left(x^{\prime}, y^{\prime}\right) \sqsubset(x, y)\right)$, then it holds that $\mathcal{R} e q_{D}\left(\mathcal{L}\left(x^{\prime}, y^{\prime}\right)\right) \subseteq \mathcal{R} e q_{D}(\mathcal{L}(x, y))$ and $\mathcal{O} b s_{D}\left(\mathcal{L}\left(x^{\prime}, y^{\prime}\right)\right) \subseteq \mathcal{R} e q_{D}(\mathcal{L}(x, y))$.

We now introduce an additional requirement on compass $\varphi$-structures stating that each temporal request is eventually fulfilled. Formally, fulfilling structures are defined as follows.

Definition 2.8. Let $\varphi$ be a $\mathrm{D}$-formula (resp., $\mathrm{D}_{\leftarrow}$-formula) and $\mathcal{G}=\left(\mathbb{P}_{\mathbb{S}}, \mathcal{L}\right)$ a compass $\varphi$-structure. We say that $\mathcal{G}$ is fulfilling if for every point $(x, y) \in \mathbb{P}_{\mathbb{S}}$ and any formula $\psi \in \mathcal{R} e q_{D}(\mathcal{L}(x, y))$, there exists a point $\left(x^{\prime}, y^{\prime}\right) \sqsubset(x, y)$ (resp., $\left.\left(x^{\prime}, y^{\prime}\right) \sqsubset(x, y)\right)$ in $\mathbb{P}_{\mathbb{S}}$ such that $\psi \in \mathcal{L}\left(x^{\prime}, y^{\prime}\right)$.

It is worth pointing out that if $\mathcal{G}$ is fulfilling, then $\mathcal{R} e q_{D}(\mathcal{L}(x, x))=\emptyset$ for all points "on the diagonal" $(x, x) \in \mathbb{P}_{\mathbb{S}}$ (corresponding to the singleton intervals of $\mathbb{I}(\mathbb{S})$ ).

As proved by Proposition 2.9 below, the fulfillment requirement ensures that, for each point $(x, y)$, the atom $\mathcal{L}(x, y)$ represents the set of formulas in $\operatorname{CL}(\varphi)$ that hold over the interval $[x, y]$ of the underlying interval model $\mathbf{M}(\mathcal{G})$.

We say that a compass $\varphi$-structure $\mathcal{G}=\left(\mathbb{P}_{\mathbb{S}}, \mathcal{L}\right)$ features a formula $\psi$ if there exists a point $(x, y) \in \mathbb{P}_{\mathbb{S}}$ such that $\psi \in \mathcal{L}(x, y)$.

The next proposition provides a characterization of the set of satisfiable D-formulas.

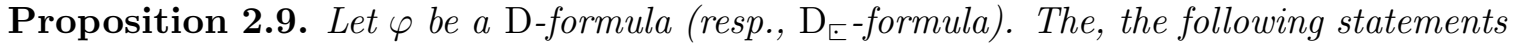
hold:

(1) given a fulfilling compass $\varphi$-structure $\mathcal{G}=\left(\mathbb{P}_{\mathbb{S}}, \mathcal{L}\right)$, it holds that for all points $(x, y)$ of $\mathcal{G}$ and $\psi \in \mathrm{CL}(\varphi), \psi \in \mathcal{L}(x, y)$ if and only if $\mathbf{M}(\mathcal{G}),[x, y] \models \psi$;

(2) $\varphi$ is satisfiable if and only if there is a fulfilling compass $\varphi$-structure that features it.

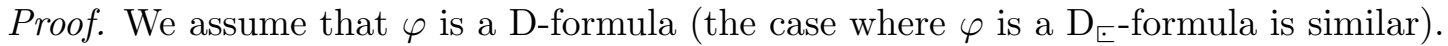

We first prove statement (1) by induction on the structure of the formula $\psi \in \mathrm{CL}(\varphi)$. The base case ( $\psi$ is a proposition letter) directly follows from the definition of $\mathbf{M}(\mathcal{G})$. The cases of the Boolean connectives follow from the induction hypothesis and the definition of $\varphi$-atoms. It remains to consider the case where $\psi$ is of the form $\langle D\rangle \psi^{\prime}$. If $\psi \in \mathcal{L}(x, y)$, then, being $\mathcal{G}$ fulfilling, there exists a point $\left(x^{\prime}, y^{\prime}\right)$ such that $\left(x^{\prime}, y^{\prime}\right) \sqsubset(x, y)$ and $\psi^{\prime} \in \mathcal{L}\left(x^{\prime}, y^{\prime}\right)$. By the induction hypothesis, it follows that $\mathbf{M}(\mathcal{G}),\left[x^{\prime}, y^{\prime}\right] \models \psi^{\prime}$, hence, $\mathbf{M}(\mathcal{G}),[x, y] \models \psi$. As for the opposite direction, assume that $\mathbf{M}(\mathcal{G}),[x, y] \models \psi$. Hence, $\mathbf{M}(\mathcal{G}),\left[x^{\prime}, y^{\prime}\right]=\psi^{\prime}$ for some interval $\left[x^{\prime}, y^{\prime}\right]$ such that $\left[x^{\prime}, y^{\prime}\right] \sqsubset[x, y]$. By the induction hypothesis, $\psi^{\prime} \in \mathcal{L}\left(x^{\prime}, y^{\prime}\right)$; by Lemma 2.7, we obtain that $\psi \in \mathcal{L}(x, y)$. 
Let us prove now statement (2). First, assume that $\varphi$ is satisfiable. Hence, there exists an interval model $\mathbf{M}$ over $\mathbb{I}(\mathbb{S})$ and an interval $[x, y] \in \mathbb{I}(\mathbb{S})$ such that $\mathbf{M},[x, y] \models \varphi$. Let $\mathcal{G}=\left(\mathbb{P}_{\mathbb{S}}, \mathcal{L}\right)$ be the weak compass $\varphi$-structure where for all points $(x, y), \mathcal{L}(x, y)$ is the set of formulas $\psi \in \mathrm{CL}(\varphi)$ such that $\mathbf{M},[x, y] \models \psi$. Since $\mathbf{M},[x, y] \models \varphi$, by the semantics of $\mathrm{D}$, it follows that $\mathcal{G}$ is a fulfilling compass $\varphi$-structure that features $\varphi$. The opposite direction directly follows from statement (1).

The notion of homogeneous models directly transfers to compass structures.

Definition 2.10. A compass $\varphi$-structure $\mathcal{G}=\left(\mathbb{P}_{\mathbb{S}}, \mathcal{L}\right)$ is homogeneous if for every point $(x, y) \in \mathbb{P}_{\mathbb{S}}$ and any $p \in \mathcal{A P}, p \in \mathcal{L}(x, y)$ if and only if $p \in \mathcal{L}\left(x^{\prime}, x^{\prime}\right)$ for all $x^{\prime} \in[x, y]$.

Proposition 2.9 (item 2) can be tailored to homogeneous compass structures as follows.

Proposition 2.11. A D $\left.\right|_{\mathcal{H} o m-f o r m u l a}$ (resp., $\left.\mathrm{D}\right|_{\mathcal{H} o m, \sqsubset-\text {-formula) }} \varphi$ is satisfiable if and only if there is a fulfilling homogeneous compass $\varphi$-structure that features it.

\section{Satisfiability of $\left.\mathrm{D}\right|_{\mathcal{H} o m}$ AND $\left.\mathrm{D}\right|_{\mathcal{H} \text { om, } 匚 \text { - }}$ OVER finite linear orders}

In this section, we devise a satisfiability checking procedure for $\left.\mathrm{D}\right|_{\mathcal{H} \text { om }}$-formulas over finite linear orders, which will also allow us to easily derive a model checking algorithm for $\left.\mathrm{D}\right|_{\mathcal{H} o m}$ over finite Kripke structures (see Section 4). At the end of this section (see Subsection 3.4), we show how to adapt the proposed approach for $\left.\mathrm{D}\right|_{\mathcal{H} o m}$ in order to obtain a decision procedure for satisfiability of $\left.\mathrm{D}\right|_{\mathcal{H} o m, ⿷}$ over finite linear orders.

In the following, we fix a $\left.\mathrm{D}\right|_{\mathcal{H} o m}$-formula $\varphi$. We first introduce a ternary relation among $\varphi$-atoms, that we denote by $=D_{\varphi} \Rightarrow$, such that if it holds among all atoms in consecutive positions of a weak compass $\varphi$-structure, then the structure is homogeneous and satisfies both the temporal consistency requirement and the fulfilling one. Hence, we may say that $=D_{\varphi} \rightarrow$ is the rule for labeling fulfilling compasses.

Next, we introduce an equivalence relation $\sim$ between rows of a compass $\varphi$-structure. Since it has finite index - exponentially bounded by $|\varphi|$ - and it preserves fulfillment of compasses, it makes it possible to "contract" the structures when we identify two related rows. Moreover, any contraction done according to $\sim$ keeps the same atoms (only the number of their occurrences may vary), and thus if a compass features $\varphi$ before the contraction, then $\varphi$ is still featured after it. This fact is exploited to build a satisfiability checking algorithm for $\left.\mathrm{D}\right|_{\mathcal{H} o m}$-formulas which makes use of polynomial working space only, because $(i)$ it only needs to keep track of two rows of a compass at a time, (ii) all rows satisfy some nice properties that allow one to succinctly encode them, and (iii) compass contractions are implicitly done by means of a reachability check in a suitable graph, whose nodes are the minimal representatives of the equivalence classes of $\sim$.

3.1. Labeling of homogeneous fulfilling compasses. We first show how to label homogeneous fulfilling compass $\varphi$-structures. Such a labeling is based on the aforementioned ternary relation $=D_{\varphi} \Rightarrow$ among atoms, which is defined as follows.

Definition 3.1. Given three $\varphi$-atoms $A_{1}, A_{2}$ and $A_{3}$, we say that $A_{3}$ is $D_{\varphi}$-generated by $A_{1}, A_{2}$ (written $A_{1} A_{2}=D_{\varphi} \Rightarrow A_{3}$ ) if:

- $A_{3} \cap \mathscr{A P}=A_{1} \cap A_{2} \cap \mathscr{A P}$ and

- $\operatorname{Req}_{D}\left(A_{3}\right)=\mathcal{R} e q_{D}\left(A_{1}\right) \cup \mathcal{R} e q_{D}\left(A_{2}\right) \cup \mathcal{O} b s_{D}\left(A_{1}\right) \cup \mathcal{O} b s_{D}\left(A_{2}\right)$. 


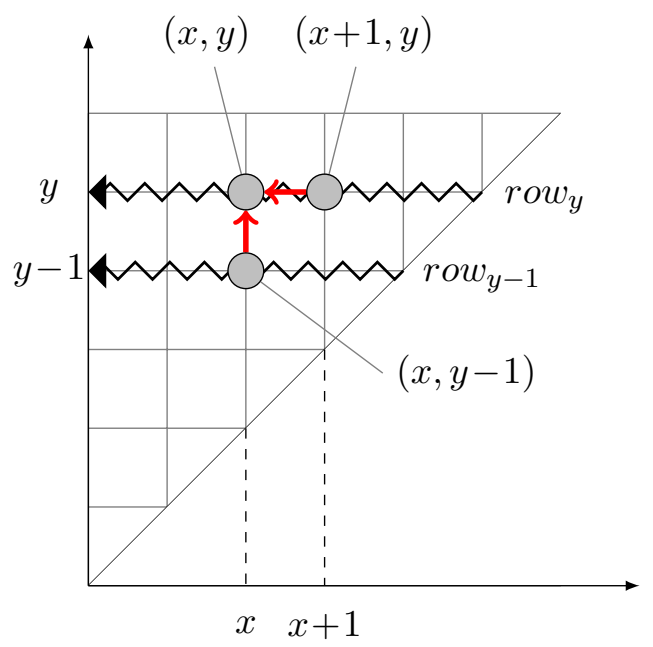

Figure 3: Rule for labeling homogeneous fulfilling compass $\varphi$-structures.

It is immediate to show that $A_{1} A_{2}=D_{\varphi} \Rightarrow A_{3}$ iff $A_{2} A_{1}=D_{\varphi} \Rightarrow A_{3}$ (i.e., the order of the first two components in the ternary relation is irrelevant). Intuitively, the first item of the definition enforces the homogeneity assumption.

The next result, which immediately follows from Proposition 2.4, proves that $=D_{\varphi} \rightarrow$ expresses a functional dependency on $\varphi$-atoms.

Lemma 3.2. Given two $\varphi$-atoms $A_{1}, A_{2} \in \mathcal{A}_{\varphi}$, there exists exactly one $\varphi$-atom $A_{3} \in \mathcal{A}_{\varphi}$ such that $A_{1} A_{2}=D_{\varphi} \Rightarrow A_{3}$.

Definition 3.1 and Lemma 3.2 can be exploited to label a homogeneous compass $\varphi$ structure $\mathcal{G}$, namely, to determine the $\varphi$-atoms labeling all the points $(x, y)$ of $\mathcal{G}$, starting from the ones on the diagonal. The idea is the following: if two $\varphi$-atoms $A_{1}$ and $A_{2}$ label respectively the greatest proper prefix $[x, y-1]$ and the greatest proper suffix $[x+1, y]$ of the same non-singleton interval $[x, y]$, then the atom $A_{3}$ labeling point $(x, y)$ is unique, and it is precisely the one satisfying $A_{1} A_{2}=D_{\varphi} \Rightarrow A_{3}$ (see Figure 3 ). The next lemma proves that this is the general rule for labeling homogeneous fulfilling compasses.

Lemma 3.3. Let $\mathcal{G}=\left(\mathbb{P}_{\mathbb{S}}, \mathcal{L}\right)$ be a weak compass $\varphi$-structure. Then, $\mathcal{G}$ is a homogeneous fulfilling compass $\varphi$-structure if and only if for each point $(x, y) \in \mathbb{P}_{\mathbb{S}}$, it holds that $(i) \mathcal{L}(x, y-$ 1) $\mathcal{L}(x+1, y)=D_{\varphi} \rightarrow \mathcal{L}(x, y)$ if $x<y$, and $($ ii $) \mathcal{R} e q_{D}(\mathcal{L}(x, y))=\emptyset$ if $x=y$.

Proof. $(\Rightarrow)$ Let us consider a point $(x, y) \in \mathbb{P}_{\mathbb{S}}$. First, we note that, since $\mathcal{G}$ is fulfilling, it must be $\mathcal{R} e q_{D}(\mathcal{L}(x, y))=\emptyset$ whenever $x=y$. Otherwise, if $x<y$, we consider the labelings $\mathcal{L}(x, y-1)$ and $\mathcal{L}(x+1, y)$. By the homogeneity property of Definition 2.10, $\mathcal{L}(x, y) \cap \mathcal{A P}=\mathcal{L}(x, y-1) \cap \mathcal{L}(x+1, y) \cap \mathscr{A P}$, and thus condition $(i)$ of Definition 3.1 holds. Moreover, since $\mathcal{G}$ is fulfilling, for every $\psi \in \mathcal{R} e q_{D}(\mathcal{L}(x, y))$ we have that either $\psi \in \mathcal{L}(x, y-1)$, or $\psi \in \mathcal{L}(x+1, y)$, or $\psi \in \mathcal{L}\left(x^{\prime}, y^{\prime}\right)$ for some $x<x^{\prime} \leq y^{\prime}<y$. In the first two cases, $\psi \in \mathcal{O} b s_{D}(\mathcal{L}(x, y-1)) \cup \mathcal{O} b s_{D}(\mathcal{L}(x+1, y))$. As for the last case, by Lemma 2.7, $\mathcal{O} b s_{D}\left(\mathcal{L}\left(x^{\prime}, y^{\prime}\right)\right) \subseteq \mathcal{R} e q_{D}(\mathcal{L}(x, y-1))$ and $\mathcal{O} b s_{D}\left(\mathcal{L}\left(x^{\prime}, y^{\prime}\right)\right) \subseteq \mathcal{R} e q_{D}(\mathcal{L}(x+1, y))$, hence $\psi \in \mathcal{R} e q_{D}(\mathcal{L}(x, y-1))$ and $\psi \in \mathcal{R} e q_{D}(\mathcal{L}(x+1, y))$. We can conclude that $\mathcal{R} e q_{D}(\mathcal{L}(x, y)) \subseteq$ $\mathcal{O} b s_{D}(\mathcal{L}(x, y-1)) \cup \mathcal{O} b s_{D}(\mathcal{L}(x+1, y)) \cup \mathcal{R} e q_{D}(\mathcal{L}(x, y-1)) \cup \mathcal{R} e q_{D}(\mathcal{L}(x+1, y))$. The converse 
inclusion $(\supseteq)$ follows by Lemma 2.7, hence condition $(i i)$ of Definition 3.1 holds. This allows us to conclude that $\mathcal{L}(x, y-1) \mathcal{L}(x+1, y)=D_{\varphi} \Rightarrow \mathcal{L}(x, y)$.

$(\Leftarrow)$ Let us consider a weak compass $\varphi$-structure $\mathcal{G}=\left(\mathbb{P}_{\mathbb{S}}, \mathcal{L}\right)$ such that for every point $(x, y) \in \mathbb{P}_{\mathbb{S}}$, we have $\mathcal{L}(x, y-1) \mathcal{L}(x+1, y)=D_{\varphi} \rightarrow \mathcal{L}(x, y)$ if $x<y$, and $\mathcal{R}_{e} q_{D}(\mathcal{L}(x, y))=\emptyset$ if $x=y$. We have to prove that $\mathcal{G}$ is a homogeneous fulfilling compass $\varphi$-structure.

First, we prove consistency with respect to the relation $D_{\varphi}$. Let us show that for all pairs of points $(x, y)$ and $\left(x^{\prime}, y^{\prime}\right)$ with $\left(x^{\prime}, y^{\prime}\right) \sqsubset(x, y)$, we have $\mathcal{L}(x, y) D_{\varphi} \mathcal{L}\left(x^{\prime}, y^{\prime}\right)$. The proof is by induction on $\Delta=\left(x^{\prime}-x\right)+\left(y-y^{\prime}\right) \geq 1$. If $\Delta=1$, either $\left(x^{\prime}, y^{\prime}\right)=$ $(x+1, y)$ or $\left(x^{\prime}, y^{\prime}\right)=(x, y-1)$. Let us consider $\left(x^{\prime}, y^{\prime}\right)=(x+1, y)$ (the other case is symmetric). Since $\mathcal{L}(x, y-1) \mathcal{L}(x+1, y)=D_{\varphi} \Rightarrow \mathcal{L}(x, y)$, by Proposition 2.5 and condition $(i i)$ of Definition 3.1, we get that $\mathcal{L}(x, y) D_{\varphi} \mathcal{L}(x+1, y)$. If $\Delta \geq 2$, since $\left(x^{\prime}, y^{\prime}\right) \sqsubset(x, y)$, then $\left(x^{\prime}, y^{\prime}+1\right) \sqsubset(x, y)$ or $\left(x^{\prime}-1, y^{\prime}\right) \sqsubset(x, y)$. We only consider $\left(x^{\prime}-1, y^{\prime}\right) \sqsubset(x, y)$, being the other case symmetric. By the inductive hypothesis, $\mathcal{L}(x, y) D_{\varphi} \mathcal{L}\left(x^{\prime}-1, y^{\prime}\right)$. Since $\mathcal{L}\left(x^{\prime}-1, y^{\prime}-1\right) \mathcal{L}\left(x^{\prime}, y^{\prime}\right)=D_{\varphi} \Rightarrow \mathcal{L}\left(x^{\prime}-1, y^{\prime}\right)$, it holds that $\mathcal{L}\left(x^{\prime}-1, y^{\prime}\right) D_{\varphi} \mathcal{L}\left(x^{\prime}, y^{\prime}\right)$. Being $D_{\varphi}$ a transitive relation, we can conclude that $\mathcal{L}(x, y) D_{\varphi} \mathcal{L}\left(x^{\prime}, y^{\prime}\right)$.

Let us now show that $\mathcal{G}$ is fulfilling. We need to prove that for every point $(x, y) \in \mathbb{P}_{\mathbb{S}}$ and for every $\psi \in \mathcal{R} e q_{D}(\mathcal{L}(x, y))$, there exists $\left(x^{\prime}, y^{\prime}\right) \in \mathbb{P}_{\mathbb{S}}$ such that $\left(x^{\prime}, y^{\prime}\right) \sqsubset(x, y)$ and $\psi \in \mathcal{L}\left(x^{\prime}, y^{\prime}\right)$. The proof is by induction on $y-x \geq 0$. If $x=y$, we have $\mathcal{R} e q_{D}(\mathcal{L}(x, y))=\emptyset$, hence the thesis holds vacuously. If $y-x \geq 1$, since $\mathcal{L}(x, y-1) \mathcal{L}(x+1, y)=D_{\varphi} \rightarrow \mathcal{L}(x, y)$, we have $\mathcal{R} e q_{D}(\mathcal{L}(x, y))=\mathcal{R} e q_{D}(\mathcal{L}(x, y-1)) \cup \mathcal{R} e q_{D}(\mathcal{L}(x+1, y)) \cup \mathcal{O} b s_{D}(\mathcal{L}(x, y-1)) \cup \mathcal{O} b s_{D}(\mathcal{L}(x+1, y))$. If $\psi \in \mathcal{O} b s_{D}(\mathcal{L}(x, y-1)) \cup \mathcal{O} b s_{D}(\mathcal{L}(x+1, y))$, the thesis is verified. If $\psi \in \mathcal{R} e q_{D}(\mathcal{L}(x+1, y))$ (the case $\psi \in \mathcal{R} e q_{D}(\mathcal{L}(x, y-1))$ is symmetric and thus omitted), by the inductive hypothesis, $\psi \in \mathcal{L}\left(x^{\prime \prime}, y^{\prime \prime}\right)$ for some $\left(x^{\prime \prime}, y^{\prime \prime}\right) \sqsubset(x+1, y) \sqsubset(x, y)$.

It remains to prove that $\mathcal{G}$ is homogeneous. We have to show that for every $(x, y) \in \mathbb{P}_{\mathbb{S}}$, $\mathcal{L}(x, y) \cap \mathcal{A P}=\bigcap_{x^{\prime} \in[x, y]} \mathcal{L}\left(x^{\prime}, x^{\prime}\right) \cap \mathscr{A P}$. The proof is by induction on $y-x \geq 0$. If $x=y$, the property trivially holds. Let us assume now $y-x>0$ (inductive step). Since $\mathcal{L}(x+1, y) \mathcal{L}(x, y-1)=D_{\varphi} \Rightarrow \mathcal{L}(x, y)$, by condition $(i)$ of Definition 3.1 and the induction hypothesis, we obtain that $\mathcal{L}(x, y) \cap \mathcal{A P}=\bigcap_{x^{\prime} \in[x+1, y]} \mathcal{L}\left(x^{\prime}, x^{\prime}\right) \cap \bigcap_{x^{\prime} \in[x, y-1]} \mathcal{L}\left(x^{\prime}, x^{\prime}\right) \cap \mathcal{A P}$. Hence, the result directly follows.

3.2. The contraction method. In this section, we describe the proposed contraction method. To begin with, we introduce the notion of $\varphi$-row, which can be viewed as the ordered sequence of (the occurrences of) atoms labelling a row of a compass $\varphi$-structure.

For a non-empty finite word (or sequence) $w$ over some finite alphabet $\Sigma$, we denote by $|w|$ the length of $w$. Moreover, for all $0 \leq i<|w|, w[i]$ denotes the $(i+1)^{t h}$ letter of $w$.

Given two non-empty finite words $w, w^{\prime}$ over $\Sigma$, we denote by $w \cdot w^{\prime}$ the concatenation of $w$ and $w^{\prime}$. If the last letter of $w$ coincides with the first letter of $w^{\prime}$, we denote by $w \star w^{\prime}$ the word $w \cdot w^{\prime}[1] \ldots w^{\prime}[n-1]$, where $n=\left|w^{\prime}\right|$, that is, the word obtained by concatenating $w$ with the word obtained from $w^{\prime}$ by erasing the first letter. When $\left|w^{\prime}\right|=1, w \star w^{\prime}=w$.

Definition 3.4. A $\varphi$-row row is a non-empty finite sequence of $\varphi$-atoms such that for all $0 \leq i<|\operatorname{row}|-1$, row $[i+1] D_{\varphi} \operatorname{row}[i]$ and $(\operatorname{row}[i] \cap \mathcal{A P}) \supseteq(\operatorname{row}[i+1] \cap \mathcal{A P})$. We say that the $\varphi$-row row is initialized if $\mathcal{R} e q_{D}($ row $[0])=\emptyset$. We represent a $\varphi$-row row in the form row $=A_{0}^{m_{0}} \cdots A_{n}^{m_{n}}$ (maximal factorization), where $A^{m}$ stands for $m$ repetitions of the $\varphi$-atom $A, m_{i}>0$ for all $i \in[0, n]$, and $A_{i} \neq A_{i+1}$ for all $i \in[0, n-1]$. 
Let $\mathcal{R o w s}_{\varphi}$ be the set of all possible $\varphi$-rows. This set is infinite. The next lemma proves that the number of distinct atoms in any $\varphi$-row row $=A_{0}^{m_{0}} \cdots A_{n}^{m_{n}}$ is linearly bounded in the size of $\varphi$.

Lemma 3.5. The number of distinct atoms in a $\varphi$-row row is at most $2|\varphi|$. Moreover, if $A_{0}^{m_{0}} \cdots A_{n}^{m_{n}}$ is the maximal factorization of row, then $A_{0}, \ldots, A_{n}$ are pairwise distinct.

Proof. First of all, since for each $0 \leq i<n, A_{i+1} D_{\varphi} A_{i}$, it holds that $\operatorname{Req}_{D}\left(A_{i}\right) \subseteq$ $\mathcal{R} e q_{D}\left(A_{i+1}\right)$. Therefore, two monotonic sequences can be associated with every $\varphi$-row, one increasing $\left(\mathcal{R} e q_{D}\left(A_{0}\right) \subseteq \mathcal{R} e q_{D}\left(A_{1}\right) \subseteq \ldots \subseteq \mathcal{R} e q_{D}\left(A_{n}\right)\right)$, and one decreasing $\left(\left(A_{0} \cap \mathcal{A P}\right) \supseteq\right.$ $\left.\left(A_{1} \cap \mathscr{A P}\right) \supseteq \ldots \supseteq\left(A_{n} \cap \mathcal{A P}\right)\right)$. The number of distinct elements is bounded by $|\varphi|$ in the former sequence and by $|\varphi|+1$ in the latter, as $\left|\operatorname{REQ}_{\varphi}\right| \leq|\varphi|-1$ and $|\mathcal{A P}| \leq|\varphi|$, since, w.l.o.g., we can restrict ourselves to the proposition letters actually occurring in $\varphi$. Given that, as already shown (Proposition 2.4), a set of requests and a set of proposition letters uniquely determine a $\varphi$-atom, any $\varphi$-row may feature at most $2|\varphi|$ distinct atoms, that is, $n \leq 2|\varphi|$. The proof of the second statement is immediate.

Given a homogeneous compass $\varphi$-structure $\mathcal{G}=\left(\mathbb{P}_{\mathbb{S}}, \mathcal{L}\right)($ for $\mathbb{S}=(S,<))$, for every $y \in S$, we define $\operatorname{row}_{y}$ as the word of $\varphi$-atoms $\operatorname{row}_{y}=\mathcal{L}(y, y) \cdots \mathcal{L}(0, y)$, that is, the sequence of atoms labeling points of $\mathcal{G}$ with the same $y$-coordinate, starting from the one on the diagonal inwards (see Figure 3). Since in a fulfilling compass $\varphi$-structure there are no temporal requests in the atoms labeling the diagonal points, we obtain the following result.

Lemma 3.6. Let $\mathcal{G}=\left(\mathbb{P}_{\mathbb{S}}, \mathcal{L}\right)$ (for $\mathbb{S}=(S,<)$ ) be a homogeneous fulfilling compass $\varphi$ structure. For every $y \in S$, row is an initialized $\varphi$-row.

We now define the successor function over $\varphi$-rows, which, given a $\varphi$-row row and a $\varphi$-atom $A$, returns the $\varphi$-row of length $\mid$ row $\mid+1$ and first atom $A$ obtained by a componentwise application of $=D_{\varphi} \Rightarrow$ starting from $A$ and the first atom of row.

Definition 3.7. Given a $\varphi$-atom $A$ and a $\varphi$-row row with $\mid$ row $\mid=n$, the $A$-successor of row, denoted by $\operatorname{succ}_{\varphi}($ row,$A)$, is the sequence $B_{0} \ldots B_{n}$ of $\varphi$-atoms defined as follows: $B_{0}=A$ and $\operatorname{row}[i] B_{i}=D_{\varphi} \Rightarrow B_{i+1}$ for all $i \in[0, n-1]$.

By Proposition 2.5 and Definition 3.1, we deduce the following lemma.

Lemma 3.8. The following properties hold:

(1) Let row be a $\varphi$-row and $A$ be a $\varphi$-atom. Then, $\operatorname{succ}_{\varphi}($ row, $A)$ is a $\varphi$-row.

(2) Let row be a $\varphi$-row of the form row $=$ row $_{1} \cdot$ row $_{2}$ and $A$ be a $\varphi$-atom. Then, $\operatorname{succ}_{\varphi}(\operatorname{row}, A)=\operatorname{succ}_{\varphi}\left(\operatorname{row}_{1}, A\right) \star \operatorname{succ}_{\varphi}\left(\operatorname{row}_{2}, A_{1}\right)$, where $A_{1}$ is the last $\varphi$-atom of $\operatorname{succ}_{\varphi}\left(\operatorname{row}_{1}, A\right)$.

Proof. Property (2) directly follows from Definition 3.7. As for Property (1), let $\operatorname{succ}_{\varphi}($ row, $A)$ $=B_{0} \ldots B_{n}$, where $n=\mid$ row $\mid$. By Definitions 3.1 and 3.7, for all $i \in[0, n-1], \mathcal{R e} q_{D}\left(B_{i}\right) \subseteq$ $\mathcal{R e q}_{D}\left(B_{i+1}\right), \mathcal{O b s}_{D}\left(B_{i}\right) \subseteq \mathcal{R} e q_{D}\left(B_{i+1}\right)$, and $\left(B_{i} \cap \mathcal{A P}\right) \supseteq\left(B_{i+1} \cap \mathcal{A P}\right)$. By Proposition 2.5, it holds that for all $i \in[0, n-1], B_{i+1} D_{\varphi} B_{i}$ and $\left(B_{i} \cap \mathcal{A P}\right) \supseteq\left(B_{i+1} \cap \mathcal{A P}\right)$. Hence, $\operatorname{succ}_{\varphi}($ row $A)$ is a $\varphi$-row.

Moreover, by Lemma 3.3, consecutive rows in homogeneous fulfilling compass $\varphi$ structures respect the successor function. In particular, the next result directly follows from Lemmata 3.3 and 3.6. 
Lemma 3.9. Let $\mathcal{G}=\left(\mathbb{P}_{\mathbb{S}}, \mathcal{L}\right)$ be a weak compass $\varphi$-structure such that $\mathcal{R} e q_{D}(\mathcal{L}(x, x))=\emptyset$ for all $(x, x) \in \mathbb{P}_{\mathbb{S}}$. Then, $\mathcal{G}$ is a homogeneous fulfilling compass $\varphi$-structure if and only if for each $0 \leq y<|S|-1$, $\operatorname{row}_{y+1}=\operatorname{succ}_{\varphi}\left(\operatorname{row}_{y}, \operatorname{row}_{y+1}[0]\right)$.

We now illustrate the kernel of the proposed approach to solve satisfiability for $\left.\mathrm{D}\right|_{\mathrm{Hom}^{-}}$ formulas. We introduce an equivalence relation $\sim$ of finite index over $\mathcal{R} o w s_{\varphi}$ whose number of classes is singly exponential in the size of $\varphi$ and such that each class has a representative whose length is polynomial in the size of $\varphi$. As a crucial result, we show that the successor function preserves the equivalence between $\varphi$-rows.

The equivalence relation $\sim$ is based on the notion of rank of $\varphi$-atoms. Given an atom $A \in \mathcal{A}_{\varphi}$, we define the $\operatorname{rank}$ of $A$, written $\operatorname{rank}(A)$, as $\left|\mathrm{REQ}_{\varphi}\right|-\left|\mathcal{R}_{e q_{D}}(A)\right|$. Clearly, $0 \leq \operatorname{rank}(A)<|\varphi|$. Whenever $A D_{\varphi} A^{\prime}$, for some $A^{\prime} \in \mathcal{A}_{\varphi}, \mathcal{R e q}_{D}\left(A^{\prime}\right) \subseteq \mathcal{R} e q_{D}(A)$, and hence $\operatorname{rank}(A) \leq \operatorname{rank}\left(A^{\prime}\right)$. We can see the rank of an atom as the "number of degrees of freedom" that it gives to the atoms that stay "above it". In particular, by Definition 3.4, for every $\varphi$-row row $=A_{0}^{m_{0}} \cdots A_{n}^{m_{n}}$, we have $\operatorname{rank}\left(A_{0}\right) \geq \cdots \geq \operatorname{rank}\left(A_{n}\right)$.

Definition 3.10. Given two $\varphi$-rows row $_{1}=A_{0}^{m_{0}} \cdots A_{n}^{m_{n}}$ and row $_{2}=\hat{A}_{0}^{\hat{m}_{0}} \cdots \hat{A}_{\hat{n}}^{\hat{m}_{\hat{n}}}$ (represented in maximal factorization form), we say that they are equivalent, written row $_{1} \sim$ row $_{2}$, if $(i) n=\hat{n}$, and $(i i)$ for each $0 \leq i \leq n, A_{i}=\hat{A}_{i}$, and either $m_{i}=\hat{m}_{i}$ or both $m_{i}$ and $\hat{m}_{i}$ are (strictly) greater than $\operatorname{rank}\left(A_{i}\right)$.

A minimal $\varphi$-row is a $\varphi$-row whose maximal factorization $A_{0}^{m_{0}} \cdots A_{n}^{m_{n}}$ satisfies the following condition: $m_{i} \in\left[1, \operatorname{rank}\left(A_{i}\right)+1\right]$, for each $0 \leq i \leq n$.

Note that if two rows feature the same set of atoms, the lower the rank of an atom $A_{i}$, the lower the number of occurrences of $A_{i}$ both the rows have to feature in order to belong to the same equivalence class. As an example, let row and $_{1}$ row $_{2}$ be two rows with $\operatorname{row}_{1}=A_{0}^{m_{0}} A_{1}^{m_{1}}, \operatorname{row}_{2}=A_{0}^{\bar{m}_{0}} A_{1}^{\bar{m}_{1}}, \operatorname{rank}\left(A_{0}\right)=4$, and $\operatorname{rank}\left(A_{1}\right)=3$. If $m_{1}=4$ and $\bar{m}_{1}=5$ they are both greater than $\operatorname{rank}\left(A_{1}\right)$, and hence they do not violate the condition for row $\sim_{1} \sim$ row $_{2}$. On the other hand, if $m_{0}=4$ and $\bar{m}_{0}=5$, we have that $m_{0}$ is less than or equal to $\operatorname{rank}\left(A_{0}\right)$. Thus, in this case, row $_{1}$ row $_{2}$ due to the indexes of $A_{0}$. This happens because $\operatorname{rank}\left(A_{0}\right)$ is greater than $\operatorname{rank}\left(A_{1}\right)$. Two cases in which $\operatorname{row}_{1} \sim \operatorname{row}_{2}$ are $m_{0}=\bar{m}_{0}$ and $m_{0}, \bar{m}_{0} \geq 5$.

The next result directly follows from the definition of $\sim$ and Lemma 3.5.

Lemma 3.11. Each equivalent class of $\sim$ contains a unique minimal $\varphi$-row. Moreover, the length of a minimal $\varphi$-row is at most $O\left(|\varphi|^{2}\right)$.

Thus, the equivalence relation $\sim$ has finite index given by the number of minimal $\varphi$-rows. This number is roughly bounded by the number of all the possible $\varphi$-rows row $=A_{0}^{m_{0}} \cdots A_{n}^{m_{n}}$, with exponents $m_{i}$ ranging from 1 to $|\varphi|$. Since $(i)$ the number of possible $\varphi$-atoms is $2^{|\varphi|}$, (ii) the number of distinct atoms in any $\varphi$-row is at most $2|\varphi|$, and (iii) the number of possible functions $f:\{1, \ldots, \ell\} \rightarrow\{1, \ldots,|\varphi|\}$ is $|\varphi|^{\ell}$, we have that the number of distinct equivalence classes of $\sim$ is bounded by

$$
\sum_{j=1}^{2|\varphi|}\left(2^{|\varphi|}\right)^{j} \cdot|\varphi|^{j} \leq 2^{3|\varphi|^{2}},
$$

which is exponential in the length of the input formula $\varphi$.

Next, we observe that if we replace a segment (sub-row) of a $\varphi$-row with an equivalent one, we obtain a $\varphi$-row which is equivalent to the original one. The following lemma holds. 
Lemma 3.12. Let row row $_{1}^{\prime}$, row row $_{2}^{\prime}$ be $\varphi$-rows such that row $\sim$ row $w_{1}^{\prime}$ and row $\sim$ row row $_{2}^{\prime}$ If row row $_{2}$ and row row $_{1}^{\prime}$ are defined, then row row $_{2} \sim$ row $_{1}^{\prime} \star$ row $_{2}^{\prime}$.

Proof. Let $A$ be the first common $\varphi$-atom $A$ of row 2 and row $w_{2}^{\prime}$ By hypothesis, $A$ is also the last common atom of row 1 and $r o w_{1}^{\prime}$. By hypothesis and Definition 3.10, one can easily deduce that, representing by $m$ (resp., $m^{\prime}$ ) the number of occurrences of $A$ in row $\star$ row row $_{2}$ (resp., row ${ }_{1}^{\prime} \star r o w_{2}^{\prime}$ ), it holds that either $m=m^{\prime}$, or both of them are greater than $\operatorname{rank}(A)$. Hence, the result easily follows.

We now show that the successor function on $\varphi$-rows preserves the equivalence of $\varphi$-rows. We first show that the result holds for $\varphi$-rows of the form $B^{m}$ for some $\varphi$-atom $B$ and $m \geq 1$.

Lemma 3.13. Let $A$ and $B$ be two $\varphi$-atoms and $m>\operatorname{rank}(B)$. Then, the following properties hold:

- the $\varphi$-row $\operatorname{succ}_{\varphi}\left(B^{m}, A\right)$ is of the form $A A_{1} \ldots A_{k}^{\ell}$ for some $k, \ell \geq 1$ such that $A_{1}, \ldots, A_{k}$ are pairwise distinct $\varphi$-atoms and $\ell>\operatorname{rank}\left(A_{k}\right)$ (note that $A$ and $A_{1}$ may be equal),

- for each $t>0, \operatorname{succ}_{\varphi}\left(B^{m+t}, A\right)=\operatorname{succ}_{\varphi}\left(B^{m}, A\right) \cdot A_{k}^{t}$.

Proof. Let row be the $\varphi$-row of length $m+1$ given by $\operatorname{succ}_{\varphi}\left(B^{m}, A\right)$. Since $\operatorname{row}[0]=A$ and $\operatorname{row}[i] B=D_{\varphi} \rightarrow \operatorname{row}[i+1]$ for all $i \in[0, m-1]$, by Definition 3.1, we easily deduce that for all $i \in[1, m]$, the following conditions hold:

- $\operatorname{row}[i] \cap \mathscr{A P}=B \cap A \cap \mathcal{A P}$;

- $\operatorname{rank}(\operatorname{row}[i-1]) \geq \operatorname{rank}(\operatorname{row}[i])$ and $\operatorname{rank}(B) \geq \operatorname{rank}(\operatorname{row}[i])$;

- if $i<m$, then $\operatorname{row}[i] \neq \operatorname{row}[i+1]$ if and only if $\operatorname{rank}\left(A_{i}\right)>\operatorname{rank}\left(A_{i+1}\right)$;

- if $i<m$ and $\operatorname{row}[i]=\operatorname{row}[i+1]$, then $\operatorname{row}[j]=\operatorname{row}[i]$ for all $j \geq i$.

Since $\operatorname{row}[0]=A$ and $\mid$ row $\mid=m+1$, it follows that row is of the form $A A_{1} \ldots A_{k}^{\ell}$ for some $k, \ell \geq 1$ such that $k+\ell-1=m, A_{1}, \ldots, A_{k}$ are pairwise distinct $\varphi$-atoms, and $\operatorname{rank}(B) \geq \operatorname{rank}\left(A_{1}\right)>\cdots>\operatorname{rank}\left(A_{k}\right)$. Hence, $\operatorname{rank}(B) \geq \operatorname{rank}\left(A_{k}\right)+k-1$. By hypothesis, $m>\operatorname{rank}(B)$ which entails that $\ell=m-k+1>\operatorname{rank}\left(A_{k}\right)$, and the first statement of Lemma 3.13 follows.

As for the second statement, we have that $\operatorname{succ}_{\varphi}\left(B^{m+t}, A\right)=\operatorname{succ}_{\varphi}\left(B^{m}, A\right) \star \operatorname{succ}_{\varphi}\left(B^{t}, A_{k}\right)$ (by Lemma 3.8(2)). If $\ell=1$, then, being $0 \leq \operatorname{rank}\left(A_{k}\right)<\ell$, it holds that $\operatorname{rank}\left(A_{k}\right)=0$. Hence, we deduce that each atom occurring in $\operatorname{succ}_{\varphi}\left(B^{t}, A_{k}\right)$ is $A_{k}$. On the other hand, if $\ell>1$ then $A_{k} B=D_{\varphi} \rightarrow A_{k}$. Hence, in both the cases, we obtain that $\operatorname{succ}_{\varphi}\left(B^{t}, A_{k}\right)$ is $A_{k}^{t+1}$ and $\operatorname{succ}_{\varphi}\left(B^{m+t}, A\right)=\operatorname{succ}_{\varphi}\left(B^{m}, A\right) \cdot A_{k}^{t}$, which concludes the proof.

We now generalize Lemma 3.13 to arbitrary $\varphi$-rows.

Lemma 3.14. Let $A$ be a $\varphi$-atom and row, row' be two $\varphi$-rows such that row $\sim$ row'. Then, it holds that $\operatorname{succ}_{\varphi}(\operatorname{row}, A) \sim \operatorname{succ}_{\varphi}\left(\operatorname{row}^{\prime}, A\right)$.

Proof. The proof is by induction on the number of distinct $\varphi$-atoms occurring in row, denoted by $N($ row $)$. Being row and row' equivalent, $N\left(\right.$ row $\left.{ }^{\prime}\right)=N($ row $)$.

Base case: $N($ row $)=N\left(\right.$ row $\left.^{\prime}\right)=1$. Assume that $\mid$ row $|\leq| r o w ' \mid$ (the case where $\left|r o w^{\prime}\right| \leq$ $\mid$ row $\mid$ being symmetric). Since row and row ${ }^{\prime}$ are equivalent, there is a $\varphi$-atom $B$ such that row $=B^{m}$, row $^{\prime}=B^{m+t}, m=\mid$ row $|, t=|$ row $|-|$ row $^{\prime} \mid$, and either $t=0$ or $m>\operatorname{rank}(B)$. If $t=0$, that is, row $=$ row $^{\prime}$, the result is obvious. Otherwise, the result directly follows from Lemma 3.13 .

Inductive step: $N($ row $)=N\left(\right.$ row $\left.^{\prime}\right)>1$. Hence, being row $\sim$ row', row (resp., row $\left.{ }^{\prime}\right)$ can be written in the form row $=$ row $_{1} \cdot$ row $_{2}$ (resp., row ${ }^{\prime}=$ row $_{1}^{\prime} \cdot$ row $_{2}^{\prime}$ ) such that 
$\operatorname{row}_{1} \sim \operatorname{row}_{1}^{\prime}$, row $_{2} \sim \operatorname{row}_{2}^{\prime}, N\left(\right.$ row $\left._{1}\right)=N\left(\right.$ row $\left._{1}^{\prime}\right)<N($ row $)=N\left(\right.$ row $\left.^{\prime}\right)$, and $N\left(\right.$ row $\left._{2}\right)=$ $N\left(\right.$ row $\left._{2}^{\prime}\right)<N($ row $)=N\left(\right.$ row $\left.^{\prime}\right)$. Let $A_{1}$ (resp., $\left.A_{1}^{\prime}\right)$ be the last atom in $\operatorname{succ}_{\varphi}\left(\operatorname{row}_{1}, A\right)$ (resp., $\left.\operatorname{succ}_{\varphi}\left(\operatorname{row}_{1}^{\prime}, A\right)\right)$. By the induction hypothesis, $\operatorname{succ}_{\varphi}\left(\operatorname{row}_{1}, A\right) \sim \operatorname{succ}_{\varphi}\left(\operatorname{row}_{1}^{\prime}, A\right)$, $A_{1}=A_{1}^{\prime}$, and $\operatorname{succ}_{\varphi}\left(\operatorname{row}_{2}, A_{1}\right) \sim \operatorname{succ}_{\varphi}\left(\operatorname{row}_{2}^{\prime}, A_{1}^{\prime}\right)$. By Lemma 3.8(2), $\operatorname{succ}_{\varphi}(\operatorname{row}, A)=$ $\operatorname{succ}_{\varphi}\left(\operatorname{row}_{1}, A\right) \star \operatorname{succ}_{\varphi}\left(\operatorname{row}_{2}, A_{1}\right)$ and $\operatorname{succ}_{\varphi}\left(\operatorname{row}^{\prime}, A\right)=\operatorname{succ}_{\varphi}\left(\operatorname{row}_{1}^{\prime}, A\right) \star \operatorname{succ}_{\varphi}\left(\operatorname{row}_{2}^{\prime}, A_{1}^{\prime}\right)$. By applying Lemma 3.12, we have that $\operatorname{succ}_{\varphi}(\operatorname{row}, A) \sim \operatorname{succ}_{\varphi}\left(\operatorname{row}^{\prime}, A\right)$ proving the thesis.

3.3. A satisfiability checking procedure for $\left.\mathbf{D}\right|_{\mathcal{H} \text { om }}$. Let us now focus on the complexity of the satisfiability checking problem for a $\left.\mathrm{D}\right|_{\mathcal{H} o m}$-formula $\varphi$ over finite linear orders, which has been proved, by Proposition 2.11, to be equivalent to the problem of deciding whether there is a homogeneous fulfilling compass $\varphi$-structure that features $\varphi$. By exploiting Lemma 3.14, we reduce such a problem to a reachability problem in a finite graph with the initialized minimal $\varphi$-rows as vertices.

Definition 3.15. Let row be a minimal $\varphi$-row and $A$ an atom. We denote by $\operatorname{succ}_{\varphi}^{\min }($ row, $A)$ the unique minimal $\varphi$-row in the equivalence class of $\sim$ containing $\operatorname{succ}_{\varphi}($ row,$A)$.

We associate with formula $\varphi$ the finite graph $G_{\varphi}^{\text {min }}=\left(\mathcal{R} o w s_{\varphi}^{\text {min }}, \Rightarrow_{\varphi}^{\text {min }}\right)$ defined as:

- $\mathcal{R o w s}_{\varphi}^{\min }$ is the set of initialized minimal $\varphi$-rows;

- for all row, row $\in \mathcal{R} o w s_{\varphi}^{\min }$, row $\Rightarrow_{\varphi}^{\text {min }} \operatorname{row}^{\prime}$ iff $\operatorname{row}^{\prime}=\operatorname{succ}_{\varphi}^{\text {min }}\left(\right.$ row $\left._{\text {row }}[0]\right)$.

We now prove the main technical result of the section.

Theorem 3.16. Let $\varphi$ be a $\left.\mathrm{D}\right|_{\mathcal{H} \text { om }}$-formula. Then, there exists a homogeneous fulfilling compass $\varphi$-structure $\mathcal{G}=\left(\mathbb{P}_{\mathbb{S}}, \mathcal{L}\right)$ that features $\varphi$ if and only if there exist two initialized

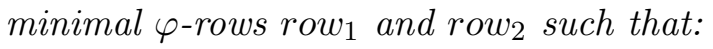

(1) $\mid$ row $_{1} \mid=1, \varphi \in$ row $_{2}[i]$ for some $0 \leq i<\mid$ row $_{2} \mid$, and

(2) row $_{2}$ is reachable from row 1 in the finite graph $G_{\varphi}^{\text {min }}=\left(\mathcal{R o w s}_{\varphi}^{\text {min }}, \Rightarrow_{\varphi}^{\text {min }}\right)$.

Proof. $(\Rightarrow)$ Let us consider a homogeneous fulfilling compass $\varphi$-structure $\mathcal{G}=\left(\mathbb{P}_{\mathbb{S}}, \mathcal{L}\right)$ that features $\varphi$. By Lemma 3.6 and Lemma 3.9, there is $m \geq 0$ and $m+1$ initialized $\varphi$-rows row $_{0}, \ldots$, row $_{m}$ such that $\mid$ row $_{0} \mid=1, \varphi \in$ row $_{m}[i]$ for some $0 \leq i<\mid$ row $_{m} \mid$, and $\operatorname{row}_{i+1}=\operatorname{succ}_{\varphi}\left(\operatorname{row}_{i}, \operatorname{row}_{i+1}[0]\right)$ for all $0 \leq i<m$.

For each $0 \leq i \leq m$, let $\operatorname{row}_{i}^{\min }$ be the unique minimal $\varphi$-row in the equivalence class $\left[\operatorname{row}_{i}\right]_{\sim}$. Note that $\operatorname{row}_{0}^{\min }=\operatorname{row}_{0}, \operatorname{row}_{i}^{\min }$ is initialized, $\operatorname{row}_{i}^{\min }[0]=\operatorname{row}_{i}[0]$, for all $i \in[0, m]$, and $\varphi \in \operatorname{row}_{m}^{\min }[j]$, for some $0 \leq j<\mid$ row $_{m}^{\min } \mid$. Moreover, by Lemma 3.14, $\operatorname{succ}_{\varphi}\left(\operatorname{row}_{i}^{\text {min }}, \operatorname{row}_{i+1}[0]\right)$ is equivalent to $\operatorname{row}_{i+1}=\operatorname{succ}_{\varphi}\left(\operatorname{row}_{i}, \operatorname{row}_{i+1}[0]\right)$, for all $0 \leq i<m$. By the definition of $\operatorname{succ}_{\varphi}^{\min }, \operatorname{row}_{i+1}^{\min }=\operatorname{succ}_{\varphi}^{\min }\left(\operatorname{row}_{i}^{\min }, \operatorname{row}_{i+1}[0]\right)$, for all $0 \leq i<m$. Hence, row ${ }_{m}^{\min }$ is reachable from row ${ }_{0}^{\text {min }}$ in the finite graph $G_{\varphi}^{\text {min }}$, and thus the thesis.

$(\Leftarrow)$ Let us assume that there exist two initialized minimal $\varphi$-rows row $_{1}$ and row $_{2}$

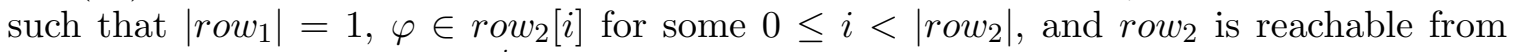
row $_{1}$ in the finite graph $G_{\varphi}^{\text {min }}$. Hence, there is $m \geq 0$ and $m+1$ initialized minimal $\varphi$-rows row $_{0}^{\text {min }}, \ldots$, row $_{m}^{\text {min }}$ such that row $_{0}^{\text {min }}=$ row $_{1}$, row $m_{m}^{\text {min }}=$ row $_{2}$, and row $_{i+1}^{\text {min }}=$ $\operatorname{succ}_{\varphi}^{\text {min }}\left(\operatorname{row}_{i}^{\text {min }}\right.$, row $\left._{i+1}^{\min }[0]\right)$, for all $0 \leq i<m$. Let row $_{0}^{\prime}, \ldots$, row $_{m}^{\prime}$ be the sequence of $\varphi$ rows defined as follows: $\operatorname{row}_{0}^{\prime}=\operatorname{row}_{0}^{\min }=\operatorname{row}_{1}$ and $\operatorname{row}_{i+1}^{\prime}=\operatorname{succ}_{\varphi}\left(\operatorname{row}_{i}^{\prime}, \operatorname{row}_{i+1}^{\min }[0]\right)$, for all $0 \leq i<m$. By applying Lemma 3.14, we deduce that $r_{0} w_{i}^{\prime} \sim \operatorname{row}_{i}^{\text {min }}$, for all $i \in[0, m]$. Hence, row $_{i}^{\prime}$ is initialized for all $i \in[0, m]$, and $\varphi \in \operatorname{row}_{m}^{\prime}[j]$, for some $0 \leq j<\left|\operatorname{row}_{m}^{\prime}\right|$. Now, let $\mathcal{G}=\left(\mathbb{P}_{\mathbb{S}}, \mathcal{L}\right)$ be a weak compass $\varphi$-structure, with $S=\{0, \ldots, m\}$ and $\mathcal{L}(x, y)=\operatorname{row}_{y}^{\prime}[y-x]$, 


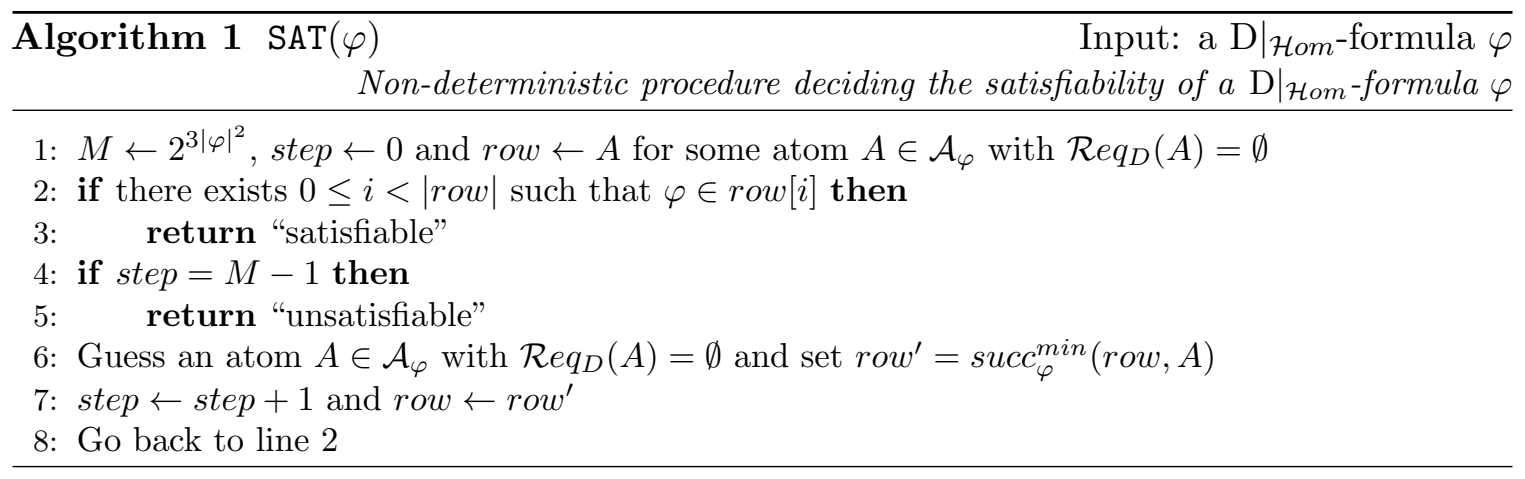

for every $0 \leq x \leq y \leq m$. By Lemma $3.9, \mathcal{G}$ is a homogeneous fulfilling compass $\varphi$-structure that features $\varphi$. This concludes the proof.

The size of $\mathcal{R o w s}_{\varphi}^{\text {min }}$ is bounded by $M=2^{3|\varphi|^{2}}$. By Theorem 3.16, $\varphi$ is satisfiable if and only if there is path in the finite graph $G_{\varphi}^{\text {min }}=\left(\mathcal{R o w s}_{\varphi}^{\min }, \Rightarrow_{\varphi}^{\text {min }}\right)$ of length at most $M$ from a $\varphi$-row in $\mathcal{R o w s}_{\varphi}^{\text {min }}$ of length 1 to a $\varphi$-row row $\in \mathcal{R}$ ows $s_{\varphi}^{\min }$ such that $\varphi \in \operatorname{row}_{2}[i]$, for some $0 \leq i<\mid$ row $_{2} \mid$. The non-deterministic procedure $\operatorname{SAT}(\varphi)$ in Algorithm 1 exploits such a characterization to decide the satisfiability of a $\left.\mathrm{D}\right|_{\mathcal{H o m}^{-}}$-formula $\varphi$. Initially, the algorithm guesses a $\varphi$-atom having no temporal request, that is, a row in $\mathcal{R o w s}_{\varphi}^{\min }$ of length 1 . At the $j$-th iteration, if the currently processed $\varphi$-row row $\in \mathcal{R}$ ows ${ }_{\varphi}^{\text {min }}$ has some atom which contains $\varphi$, then the algorithm terminates with success. Otherwise, the algorithm guesses a successor row' of the current $\varphi$-row row in $G_{\varphi}^{m i n}$. The procedure terminates after at most $M$ iterations. The working space used by the procedure is polynomial: $M$ and step (which ranges in $[0, M-1]$ ) can be encoded in binary with $\left\lceil\log _{2} M\right\rceil+1=O\left(|\varphi|^{2}\right.$ ) bits. Moreover, at each step, the algorithm keeps in memory only two minimal initialized $\varphi$ rows: the current one row and the guessed successor row in $G_{\varphi}^{\text {min }}$. By Lemma 3.11, each minimal initialized $\varphi$-row can be represented by using space polynomial in $\varphi$. Thus, since NPSPACE = PSPACE, we obtain the following result.

Theorem 3.17. The satisfiability problem for $\left.\mathrm{D}\right|_{\mathcal{H} \text { om }}$-formulas over finite linear orders is in PSPACE.

3.4. The easy adaptation to $\left.\mathbf{D}\right|_{\mathcal{H} o m, \sqsubset}$. We conclude the section by sketching the changes to the previous notions that allow us to prove the decidability of the satisfiability problem for $\left.\mathrm{D}\right|_{\mathcal{H} o m, ⿷}$ over finite linear orders. As a matter of fact, it suffices to replace the definitions of $=D_{\varphi} \Rightarrow, \varphi$-row, and $\operatorname{succ}_{\varphi}$ by the following ones. For the sake of simplicity, we introduce a dummy atom $\square$, for which we assume $\mathcal{R} e q_{D}(\bullet)=\mathcal{O} b s_{D}(\square)=\emptyset$.

Definition 3.18. Given the $\varphi$-atoms $A_{1}, A_{3}, A_{4} \in \mathcal{A}_{\varphi}$ and $A_{2} \in \mathcal{A}_{\varphi} \cup\{\boxminus\}$, we say that $A_{4}$ is $D_{\varphi}$ ⿷-generated by $A_{1}, A_{2}, A_{3}$ (written $A_{1}, A_{2}, A_{3}=D_{\varphi} \sqsubset \Rightarrow A_{4}$ ) if:

- $A_{4} \cap \mathscr{A P}=A_{1} \cap A_{3} \cap \mathscr{A P}$ and

- $\mathcal{R} e q_{D}\left(A_{4}\right)=\mathcal{R} e q_{D}\left(A_{1}\right) \cup \mathcal{R} e q_{D}\left(A_{3}\right) \cup \mathcal{O} b s_{D}\left(A_{2}\right)$.

The intuition behind Definition 3.18 is that if an interval $[x, y]$, with $x<y$, is labeled by $A_{4}$, and its three sub-intervals $[x, y-1],[x+1, y-1]$, and $[x+1, y]$ are labeled by $A_{1}, A_{2}$, and $A_{3}$, respectively, we require that $A_{1}, A_{2}, A_{3}=D_{\varphi} \sqsubset \Rightarrow A_{4}$ holds. In particular, in the special 


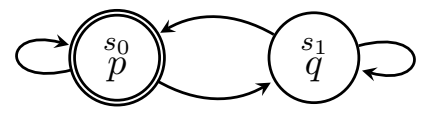

Figure 4: Kripke structure $\mathcal{K}_{2}$.

case where $x=y-1$, we have $A_{2}=\square$ since $[x+1, y-1]$ is not a valid interval. Moreover, since the only strict sub-interval is $[x+1, y-1]$ (i.e., $[x+1, y-1] \sqsubset[x, y]$ ), we require that $\mathcal{O} b s_{D}\left(A_{2}\right) \subseteq \mathcal{R} e q_{D}\left(A_{4}\right)$. Finally, since the requests of $A_{1}$ and $A_{3}$ are fulfilled by a strict sub-interval of $[x, y]$, we require that $\mathcal{R e q}_{D}\left(A_{1}\right) \subseteq \mathcal{R} e q_{D}\left(A_{4}\right)$ and $\mathcal{R} e q_{D}\left(A_{3}\right) \subseteq \mathcal{R} e q_{D}\left(A_{4}\right)$.

Definition 3.19. A $\varphi-\leftarrow$-row row is a non-empty finite sequence of $\varphi$-atoms such that for all $0 \leq i<|\operatorname{row}|-1, \mathcal{R} e q_{D}(\operatorname{row}[i]) \subseteq \mathcal{R} e q_{D}(\operatorname{row}[i+1])$ and $(\operatorname{row}[i] \cap \mathcal{A P}) \supseteq(\operatorname{row}[i+1] \cap \mathcal{A P})$. The $\varphi$-⿷-row row is initialized if $\mathcal{R} e q_{D}($ row $[0])=\emptyset$.

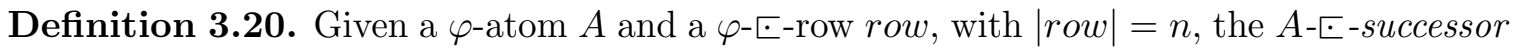
of row, denoted by $\operatorname{succ}_{\varphi, \sqsubset}($ row,$A)$, is the sequence $B_{0} \ldots B_{n}$ of $\varphi$-atoms defined as follows: $B_{0}=A$ and row $[i] \operatorname{row}[i-1] B_{i}=D_{\varphi} \rightarrow B_{i+1}$ for all $i \in[0, n-1]$, with $\operatorname{row}[i-1]=\bullet$ for $i=0$.

Once the above-defined changes have been to the basic notions, following exactly the same steps of the proof of Theorem 3.17, we can show that like $\left.\mathrm{D}\right|_{\mathcal{H} o m}$, satisfiability for $\left.\mathrm{D}\right|_{\mathcal{H o m , \Sigma}}$ over finite linear orders is in PSPACE.

Theorem 3.21. The satisfiability problem for $\left.\mathrm{D}\right|_{\mathcal{H} \text { om, } 匚 \text {-formulas over finite linear orders is }}$ in PSPACE.

PSPACE-completeness of the satisfiability problem for $\left.\mathrm{D}\right|_{\mathcal{H} o m}$ and $\left.\mathrm{D}\right|_{\mathcal{H} o m \text {, ⿷ }}$ will be proved in Section 5. In the next section, we focus on their model checking problem.

\section{Model Checking of $\left.\mathrm{D}\right|_{\mathcal{H} \text { om }}$ And $\left.\mathrm{D}\right|_{\mathcal{H} \text {, ⿷匚 }}$ FORmulas over Kripke structures}

In this section, we deal with the model checking problem for $\left.\mathrm{D}\right|_{\mathcal{H} o m}$ and $\left.\mathrm{D}\right|_{\mathcal{H} \text { om,E्E }}$, namely, the problem of checking whether a model of a given system satisfies some behavioural properties expressed as $\left.\mathrm{D}\right|_{\mathcal{H}^{\prime} m^{-}}$or $\left.\mathrm{D}\right|_{\mathcal{H} \text { om, } \text { - }^{-} \text {-formulas. The usual models are Kripke structures, which }}$ will now be introduced along with the definition of the semantics of $\left.\mathrm{D}\right|_{\mathcal{H} \text { om }}$ and $\left.\mathrm{D}\right|_{\mathcal{H} \text {, ⿷ } 匚 \text { E }}$ formulas over them.

Definition 4.1. A finite Kripke structure is a tuple $\mathcal{K}=\left(\mathscr{A} \mathscr{P}, W, E, \mu, s_{0}\right)$, where $\mathscr{A} \mathcal{P}$ is a finite set of proposition letters, $W$ is a finite set of states, $E \subseteq W \times W$ is a left-total binary relation over $W, \mu: W \rightarrow 2^{\mathfrak{A P}}$ is a labelling function over $W$, and $s_{0} \in W$ is the initial state.

For all $s \in W, \mu(s)$ is the set of proposition letters that hold on $s$, while $E$ is the transition relation that describes the evolution of the system over time.

Figure 4 depicts a finite Kripke structure $\mathcal{K}_{2}=\left(\{p, q\},\left\{s_{0}, s_{1}\right\}, E, \mu, s_{0}\right)$, with $E=$ $\left\{\left(s_{0}, s_{0}\right),\left(s_{0}, s_{1}\right),\left(s_{1}, s_{0}\right),\left(s_{1}, s_{1}\right)\right\}, \mu\left(s_{0}\right)=\{p\}$, and $\mu\left(s_{1}\right)=\{q\}$. The initial state $s_{0}$ is identified by a double circle.

Definition 4.2 (Paths and traces). Given a finite Kripke structure $\mathcal{K}=\left(\mathscr{A} \mathscr{P}, W, E, \mu, s_{0}\right)$, a path of $\mathcal{K}$ is a non-empty finite sequence of states $\rho=s_{1} \cdots s_{n}$ such that $\left(s_{i}, s_{i+1}\right) \in E$ for $i=1, \ldots, n-1$. A path is initial if it starts from the initial state of $\mathcal{K}$.

We extend the labeling $\mu$ to paths of $\mathcal{K}$ in the usual way: for a path $\rho=s_{1} \ldots s_{n}, \mu(\rho)$ denotes the word over $2^{\mathcal{A} \mathscr{P}}$ of length $n$ given by $\mu\left(s_{1}\right) \ldots \mu\left(s_{n}\right)$. A trace of $\mathcal{K}$ is a non-empty 


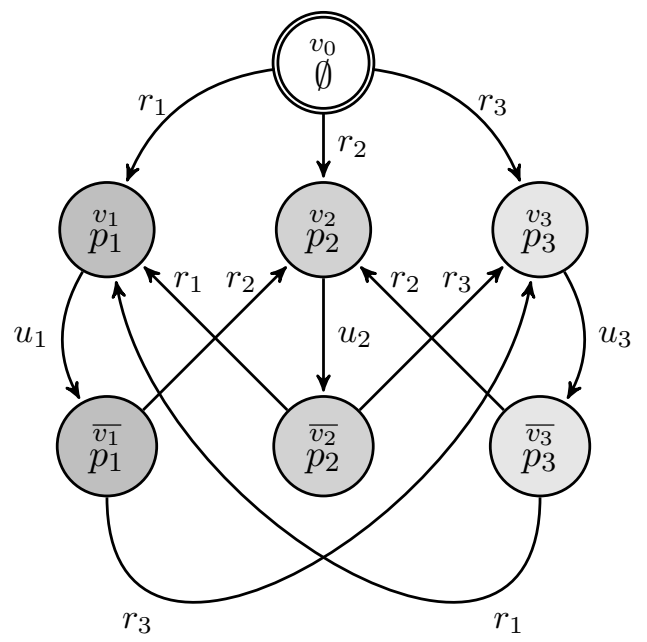

Figure 5: The Kripke structure $\mathcal{K}_{\text {Sched }}$ of Example 4.5.

finite word over $2^{\mathfrak{A P}}$ of the form $\mu(\rho)$ for some path $\rho$ of $\mathcal{K}$. A trace is initial if it is of the form $\mu(\rho)$ for some initial path $\rho$ of $\mathcal{K}$.

Given a non-empty finite word $w$ over $2^{\mathfrak{A P}}$, we can associate with $w$, in a natural way, a homogeneous interval model $\mathbf{M}(w)$ over the finite linear order induced by $w$.

Definition 4.3. For a non-empty finite word $w$ over $2^{\mathfrak{A P} P}$, the interval model $\mathbf{M}(w)=$ $\langle\mathbb{I}(\mathbb{S}), \mathcal{V}\rangle$ induced by $w$ is the homogeneous interval model defined as follows:

(1) $\mathbb{S}=\langle S,<\rangle$, where $S=\{0, \ldots,|w|-1\}$, and

(2) for every interval $[x, y] \in \mathbb{I}(\mathbb{S})$ and $p \in \mathcal{A P},[x, y] \in \mathcal{V}(p)$ if and only if $p \in w\left[x^{\prime}\right]$ for all $x^{\prime} \in[x, y]$.

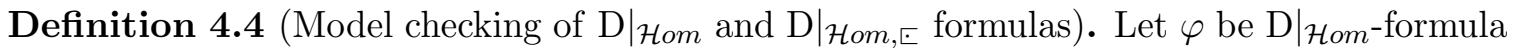
(resp., $\left.\mathrm{D}\right|_{\mathcal{H} o m, \check{E}^{-}}$formula). Given a non-empty finite word $w$ over $2^{\mathfrak{A P}}$, $w$ satisfies $\varphi$, denoted by $w=\varphi$, if $\mathbf{M}(w),[0,|w|-1] \mid=\varphi$. A finite Kripke structure $\mathcal{K}$ over $\mathcal{A P}$ is a model of the formula $\varphi$ if for each initial trace $w$ of $\mathcal{K}$, it holds that $w \models \varphi$. The model-checking $(M C)$ problem for $\left.\mathrm{D}\right|_{\mathcal{H} \text { om }}$ (resp., $\left.\mathrm{D}\right|_{\mathcal{H} \text { om, ⿷匚 }}$ ) is the problem of deciding for a given finite Kripke structure $\mathcal{K}$ and $\left.\mathrm{D}\right|_{\mathcal{H} \text { om }}$-formula (resp., $\left.\mathrm{D}\right|_{\mathcal{H} \text { om, } 匚 \text {-formula) }} \varphi$, whether $\mathcal{K}=\varphi$.

Example 4.5. In Figure 5, we give an example of a finite Kripke structure $\mathcal{K}_{S c h e d}$ that models the behaviour of a scheduler serving three processes which are continuously requesting the use of a common resource. The initial state is $v_{0}$ : no process is served in that state. In the states $v_{i}$ and $\bar{v}_{i}$, with $i \in\{1,2,3\}$, the $i$-th process is served (this is denoted by the fact that $p_{i}$ holds in those states). For the sake of readability, edges are marked either by $r_{i}$, for request $(i)$, or by $u_{i}$, for unlock $(i)$. Edge labels do not have a semantic value, that is, they are neither part of the structure definition, nor proposition letters; they are simply used to ease reference to edges. Process $i$ is served in state $v_{i}$, then, after "some time", a transition $u_{i}$ from $v_{i}$ to $\bar{v}_{i}$ is taken; subsequently, process $i$ cannot be served again immediately, as $v_{i}$ is not directly reachable from $\bar{v}_{i}$ (the scheduler cannot serve the same process twice in two successive rounds). A transition $r_{j}$, with $j \neq i$, from $\bar{v}_{i}$ to $v_{j}$ is then taken and process $j$ is served. This structure can be easily generalised to an arbitrary number of processes. 
We now show how some meaningful behavioural properties of the Kripke structure $\mathcal{K}_{\text {Sched }}$ can be expressed by $\left.\mathrm{D}\right|_{\mathcal{H} \text { om }}$-formulas.

Preliminarily, we observe that the formula $l e n_{\geq i}:=\langle D\rangle^{i-1} \top$ is satisfied by traces at least $i$ units long, and, analogously, $l e n_{\leq i}:=[D]^{i} \perp$ by traces at most $i$ units long. We define len $=i:=\operatorname{len}_{\geq i} \wedge \operatorname{len}_{\leq i}$.

In all the following formulas, we force the validity of the considered properties over all legal computation sub-intervals by using the modality $[D]$ (all computation sub-intervals are sub-intervals of at least one initial trace of the Kripke structure).

The first formula requires that "at least 2 processes are witnessed in any sub-interval of length at least 5 of an initial trace". Since a process cannot be executed twice in a row, such a formula is satisfied by $\mathcal{K}_{\text {Sched }}$ :

$$
\mathcal{K}_{\text {Sched }}=[D]\left(\text { len }_{\geq 5} \rightarrow \bigvee_{1 \leq i<j \leq 3}\left(\langle D\rangle p_{i} \wedge\langle D\rangle p_{j}\right)\right)
$$

The second formula requires that "in any sub-interval of length at least 11 of an initial trace, process 3 is executed at least once in some states" (non starvation). KSched does not satisfy it, because the scheduler can postpone the execution of a process ad libitum:

$$
\mathcal{K}_{\text {Sched }} \not \models[D]\left(l e n_{\geq 11} \rightarrow\langle D\rangle p_{3}\right) .
$$

The third formula requires that "in any sub-interval of length at least 6 of an initial trace, $p_{1}, p_{2}$, and $p_{3}$ are all witnessed". The only way to satisfy this property would be to force the scheduler to execute the three processes in a strictly periodic manner (strict alternation), that is, $p_{i} p_{j} p_{k} p_{i} p_{j} p_{k} p_{i} p_{j} p_{k} \cdots$, for $i, j, k \in\{1,2,3\}$ and $i \neq j \neq k \neq i$, but $\mathcal{K}_{\text {Sched }}$ does not meet such a requirement:

$$
\mathcal{K}_{\text {Sched }} \not \models[D]\left(\text { len }_{\geq 6} \rightarrow\left(\langle D\rangle p_{1} \wedge\langle D\rangle p_{2} \wedge\langle D\rangle p_{3}\right)\right) \text {. }
$$

Finally, we write two formulas expressing safety properties: "it is never the case that processes 1 and 2 are executed consecutively", and "it is never the case that a state where process 1 is executed, is reached". Neither of these is satisfied:

$$
\begin{gathered}
\mathcal{K}_{\text {Sched }} \not \models[D]\left(\text { len }_{=4} \rightarrow\left(\neg\langle D\rangle p_{1} \vee \neg\langle D\rangle p_{2}\right)\right), \\
\mathcal{K}_{\text {Sched }} \forall \models[D] \neg p_{1} .
\end{gathered}
$$

We now show how, by slightly modifying the satisfiability checking procedure given in Section 3, it is possible to obtain an automata-theoretic MC algorithm for $\left.\mathrm{D}\right|_{\mathcal{H} \text { om }}$-formulas over finite Kripke structures $\mathcal{K}$. Let $G_{\varphi}^{\min }=\left(\mathcal{R o w s}_{\varphi}^{\min }, \Rightarrow_{\varphi}^{\min }\right)$ be the finite graph of Definition 3.15 associated with the $\left.\mathrm{D}\right|_{\mathcal{H} o m}$-formula $\varphi$. We first show that it is possible to construct a standard deterministic finite automaton (DFA) $\tilde{\mathcal{N}}_{\varphi}$ over the alphabet $2^{\mathfrak{A P} P}$ with set of states $\mathcal{R o w s}_{\varphi}^{\min }$, which accepts all and only the non-empty finite words over $2^{\mathfrak{A P} \mathcal{P}}$ that satisfy formula $\varphi$. Next, given a finite Kripke structure $\mathcal{K}$ and a $\left.\mathrm{D}\right|_{\mathcal{H} \text { om }}$-formula $\varphi$, to check whether $\mathcal{K}$ is a model of $\varphi$, we apply the standard approach to MC taking the synchronous product of $\mathcal{K}$ with the automaton $\tilde{\mathcal{N}}_{\neg \varphi}$ for the negation of the formula $\varphi\left(\mathcal{K} \times \tilde{\mathcal{N}}_{\neg \varphi}\right.$ for short). $\mathcal{K} \times \tilde{\mathcal{N}}_{\neg \varphi}$ accepts all and only the initial traces of $\mathcal{K}$ that violate the property $\varphi$. Hence, $\mathcal{K}$ is a model of $\varphi$ if and only if the language accepted by $\mathcal{K} \times \tilde{\mathcal{N}}_{\neg \varphi}$ is empty.

We now provide the technical details.

A nondeterministic finite-state automaton (NFA) is a tuple $\mathcal{N}=\left(\Sigma, Q, q_{1}, \delta, F\right)$, where $\Sigma$ is a finite alphabet, $Q$ is a finite set of states, $q_{1} \in Q$ is the initial state, $\delta: Q \times \Sigma \rightarrow 2^{Q}$ is the transition function, and $F \subseteq Q$ is the set of accepting states. Given a finite word $w$ over 
$\Sigma$, with $|w|=n$, a computation of $\mathcal{N}$ over $w$ is a finite sequence of states $q_{1}^{\prime}, \ldots, q_{n+1}^{\prime}$ such that $q_{1}^{\prime}=q_{1}$, and for all $i \in[0, n-1], q_{i+2}^{\prime} \in \delta\left(q_{i+1}^{\prime}, w[i]\right)$. The language $\mathcal{L}(\mathcal{N})$ accepted by $\mathcal{N}$ consists of the finite words $w$ over $\Sigma$ such that there is a computation over $w$ ending in some accepting state. A deterministic finite-state automaton $(\mathrm{DFA})$ is an NFA $\tilde{\mathcal{N}}=\left(\Sigma, \tilde{Q}, \tilde{q_{1}}, \tilde{\delta}, \tilde{F}\right)$ such that for all $(q, c) \in \tilde{Q} \times \Sigma, \tilde{\delta}(q, c)$ is a singleton.

Let $\mathcal{K}=\left(\mathscr{A P}, W, E, \mu, s_{0}\right)$ be a finite Kripke structure and $\mathcal{N}=\left(2^{\mathfrak{A P}}, Q, q_{1}, \delta, F\right)$ be an NFA. The synchronous product of $\mathcal{K}$ and $\mathcal{N}$ (denoted by $\mathcal{K} \times \mathcal{N})$ is the NFA $\left(2^{\mathscr{A} P}, W \times\right.$ $\left.Q,\left(s_{0}, q_{1}\right), \delta^{\prime}, W \times F\right)$, where for all $(s, q) \in W \times Q$ and $P \in 2^{\mathfrak{A P}}, \delta^{\prime}((s, q), P)=\emptyset$ if $P \neq \mu(s)$, and $\delta^{\prime}((s, q), P)$ is the set of pairs $\left(s^{\prime}, q^{\prime}\right) \in W \times Q$ such that $\left(s, s^{\prime}\right) \in E$ and $q^{\prime} \in \delta(q, P)$ otherwise. It can be easily seen that $\mathcal{K} \times \mathcal{N}$ accepts all and only the initial traces of $\mathcal{K}$ which are accepted by $\mathcal{N}$.

Let $\varphi$ be a $\left.\mathrm{D}\right|_{\mathcal{H o m}}$-formula and $G_{\varphi}^{\text {min }}=\left(\mathcal{R o w s}_{\varphi}^{\min }, \Rightarrow_{\varphi}^{\min }\right)$ be the finite graph of Definition 3.15, where $\mathcal{R o w s}_{\varphi}^{\text {min }}$ is the set of initialized minimal $\varphi$-rows and row $\Rightarrow_{\varphi}^{\text {min }}$ row' if

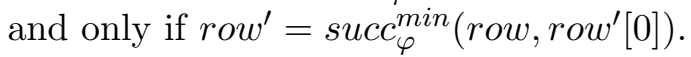

Definition 4.6. Let $P \subseteq \mathcal{A P}$ be a set of proposition letters and $\varphi$ be a $\left.\mathrm{D}\right|_{\mathcal{H} \text { om }}$-formula. We denote by $A(P)$ the unique $\varphi$-atom such that $A(P) \cap \mathcal{A P}=P$ and $\mathcal{R}_{e} q_{D}(A(P))=\emptyset$. We associate with $\varphi$ the DFA $\tilde{\mathcal{N}}_{\varphi}=\left(2^{\mathfrak{A P} P}, \mathcal{R}\right.$ ows $\left.s_{\varphi}^{\min } \cup\left\{\tilde{q_{1}}\right\}, \tilde{q_{1}}, \tilde{\delta}, \tilde{F}\right)$, where $\tilde{\delta}$ and $\tilde{F}$ are defined as follows:

- $\tilde{\delta}\left(\tilde{q_{1}}, P\right)=A(P)$, for all $P \in 2^{\mathfrak{A P}}$;

- $\tilde{\delta}($ row,$P)=\operatorname{succ}_{\varphi}^{\min }($ row,$A(P))$, for all $P \in 2^{\mathfrak{A P} P}$ and row $\in \mathcal{R} o w s_{\varphi}^{\min }$;

- $\tilde{F}$ is the set of $\varphi$-row row $\in \mathcal{R} o w s_{\varphi}^{\text {min }}$ such that $\varphi \in \operatorname{row}[n-1]$, where $n=\mid$ row $\mid$.

By exploiting Theorem 3.16, we get the following result, that outlines an automatatheoretic approach to $\mathrm{MC}$ for $\left.\mathrm{D}\right|_{\mathcal{H} o m}$.

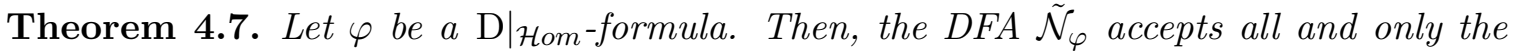
non-empty finite words over $2^{\mathcal{A P}}$ that satisfy $\varphi$.

Proof. Let $w$ be a non-empty finite word over $2^{\mathfrak{A} P}$ and $n=|w|-1$. We show that $\mathbf{M}(w),[0, n] \models \varphi$ if and only if $w \in \mathcal{L}\left(\tilde{\mathcal{N}}_{\varphi}\right)$.

$(\Rightarrow)$ Let us assume that $\mathbf{M}(w),[0, n] \models \varphi$. We define $\mathcal{G}$ as the weak compass $\varphi$ structure $\left(\mathbb{P}_{\mathbb{S}}, \mathcal{L}\right)$, where $\mathbb{S}=(\{0, \ldots, n\},<)$ and, for all points $(x, y), \mathcal{L}(x, y)$ is the set of formulas $\psi \in \operatorname{CL}(\varphi)$ such that $\mathbf{M}(w),[x, y] \mid=\psi$. By the semantics of $\left.\mathrm{D}\right|_{\mathcal{H} o m}, \mathcal{G}$ is a homogeneous fulfilling compass $\varphi$-structure. For all $i \in[0, n]$, let row $_{i}$ be the $i^{\text {th }} \varphi$-row of $\mathcal{G}$. By construction, $\varphi \in \operatorname{row}_{n}[n]$ and $\operatorname{row}_{i}[0]=A(w[i])$, for all $i \in[0, n]$. By the proof of Theorem 3.16 (right implication), there exist $n+1$ initialized minimal $\varphi$-rows row $_{0}^{\text {min }}, \ldots$, row $_{n}^{\text {min }}$ such that row ${ }_{0}^{\text {min }}=$ row $_{0}, \operatorname{row}_{i}^{\text {min }}$ is equivalent to $\operatorname{row}_{i}$, for all $i \in[0, n]$, and row $_{i+1}^{\min }=\operatorname{succ}_{\varphi}^{\text {min }}\left(\right.$ row $\left._{i}^{\text {min }}, \operatorname{row}_{i+1}^{\min }[0]\right)$, for all $i \in[0, n-1]$. It follows that the last atom of $\operatorname{row}_{n}^{\min }$ contains $\varphi$ and $\operatorname{row}_{i}^{\min }[0]=A(w[i])$, for all $i \in[0, n]$. By Definition 4.6, it follows that there is an accepting computation of $\tilde{\mathcal{N}}_{\varphi}$ over $w$, that is, $w \in \mathcal{L}\left(\tilde{\mathcal{N}}_{\varphi}\right)$.

$(\Leftarrow)$ Let us assume that $w$ is accepted by $\tilde{\mathcal{N}}_{\varphi}$. By Definition 4.6 , there are $n+1$ initialized minimal $\varphi$-rows $\operatorname{row}_{0}^{\min }, \ldots$, row $_{n}^{\text {min }}$ such that $\operatorname{row}_{0}^{\min }=A(w(0)), \varphi \in \operatorname{row}_{n}^{\min }[n]$, $\operatorname{row}_{i}^{\min }[0]=A(w(i))$, for all $0 \leq i \leq n$, and $\operatorname{row}_{i+1}^{\min }=\operatorname{succ}_{\varphi}^{\text {min }}\left(\operatorname{row}_{i}^{\text {min }}, \operatorname{row}_{i+1}^{\text {min }}[0]\right)$ for all $0 \leq i<n$. By the proof of Theorem 3.16 (left implication), there is a fulfilling homogeneous compass $\varphi$-structure $\mathcal{G}=\left(\mathbb{P}_{\mathbb{S}}, \mathcal{L}\right)$, with $S=\{0, \ldots, n\}$, such that, for all $0 \leq j<n$, its

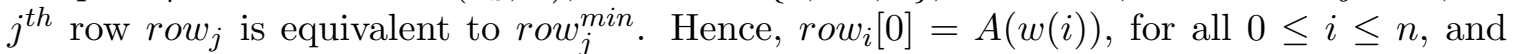
the last atom of row $_{n}$ contains $\varphi$. Since in a homogeneous interval model the valuation 
function is completely specified by the values taken at the singleton intervals, it follows that $\mathbf{M}(\mathcal{G})=\mathbf{M}(w)$. Moreover, by Proposition 2.9, $\mathbf{M}(\mathcal{G}),[0, n] \models \varphi$. Hence, $\mathbf{M}(w),[0, n] \models \varphi$ and the thesis follows.

By Theorem 4.7, we get the main result of the section.

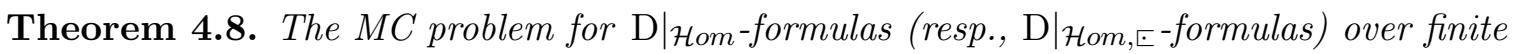
linear orders is in PSPACE. For constant-length formulas, it is in NLOGSPACE.

Proof. By Theorem 4.7, given a finite Kripke structure $\mathcal{K}$ and a $\left.\mathrm{D}\right|_{\mathcal{H} \text { om }}$-formula $\varphi, \mathcal{K} \not \models \varphi$ if and only if the language accepted by $\mathcal{K} \times \tilde{\mathcal{N}}_{\neg \varphi}$ is not empty. Similarly to Algorithm 1 of Section 3, the problem of establishing whether $\mathcal{L}\left(\mathcal{K} \times \tilde{\mathcal{N}}_{\neg \varphi}\right) \neq \emptyset$ can be solved by a nondeterministic algorithm which uses space logarithmic in the number of states of $\mathcal{K} \times \tilde{\mathcal{N}}_{\neg \varphi}$ and checks whether some accepting state is reachable from the initial one. Since the number of states in $\mathcal{K} \times \tilde{\mathcal{N}}_{\neg \varphi}$ is linear in the number of states of $\mathcal{K}$ and singly exponential in the length of $\varphi$, and the complexity classes NPSPACE = PSPACE and NLOGSPACE are closed under complementation, the result for $\left.\mathrm{D}\right|_{\mathcal{H} \text { om }}$ directly follows. The MC procedure for $\left.\mathrm{D}\right|_{\mathcal{H} \text { om }}$ can be easily adapted to $\left.\mathrm{D}\right|_{\mathcal{H} \text { om, } 匚 \text { - }}$ by making use of Definitions 3.18-3.20.

In the next section, we will prove that $\mathrm{MC}$ for $\left.\mathrm{D}\right|_{\mathcal{H} o m}$-formulas and $\left.\mathrm{D}\right|_{\mathcal{H} o m, ⿷}$-formulas is PSPACE-hard.

5. Hardness of MC and satisfiability Checking of $\left.\mathrm{D}\right|_{\mathcal{H} \text { om }}$ And $\left.\mathrm{D}\right|_{\mathcal{H} \text { hom,E }}$ Formulas OVER FINITE LINEAR ORDERS

In this section, we provide lower bounds for $\mathrm{MC}$ and satisfiability checking for $\left.\mathrm{D}\right|_{\mathcal{H} o m}$ (resp., $\left.\mathrm{D}\right|_{\mathcal{H} \text { om, } 匚}$ ) over finite linear orders that match the upper bounds of Theorem 4.8 and Theorem 3.17 (resp., Theorem 3.21).

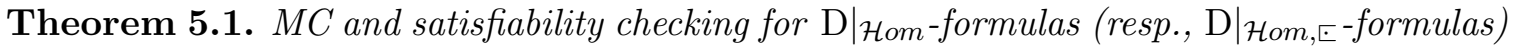
over finite linear orders are both $\boldsymbol{P S P A C E}$-hard.

By a trivial reduction from the problem of (non-)reachability of two nodes in a directed graph, it easily follows that $\mathrm{MC}$ for constant-length $\left.\mathrm{D}\right|_{\mathcal{H} o m}$-formulas (resp., $\left.\mathrm{D}\right|_{\mathcal{H} \text { om, } \mathbf{E}^{-}}$ formulas) is NLOGSPACE-hard. By taking into account the upper bounds given by Theorems 4.8, 3.17, and 3.21, we obtain the following corollary.

Corollary 5.2. $M C$ and satisfiability checking for $\left.\mathrm{D}\right|_{\mathcal{H} o m}$-formulas (resp., $\left.\mathrm{D}\right|_{\mathcal{H} \text { Hom, ⿷- }- \text { formulas) }}$ over finite linear orders are both PSPACE-complete. Moreover, when the length of the formula is fixed, MC is NLOGSPACE-complete.

The rest of the section is devoted to the proof of Theorem 5.1. We focus on $\left.\mathrm{D}\right|_{\mathcal{H} \text { om }}$. The proof for $\left.\mathrm{D}\right|_{\mathcal{H} \text { om,⿷匚 }}$ is very similar, and thus we omit it.

We prove Theorem 5.1 by means of a polynomial time reduction from a domino-tiling problem for grids with rows of linear length [Har92]. To start with, we fix an instance $\mathcal{I}$ of such a problem, which is a tuple $\mathcal{I}=\left(C, \Delta, n, d_{\text {init }}, d_{\text {final }}\right)$, where $C$ is a finite set of colors, $\Delta \subseteq C^{4}$ is a set of tuples $\left(c_{\text {down }}, c_{\text {left }}, c_{\text {up }}, c_{\text {right }}\right)$ of four colors, called domino-types, $n>1$ is a natural number encoded in unary, and $d_{\text {init }}, d_{\text {final }} \in \Delta$ are two distinguished domino-types (respectively, the initial and final ones). A grid of $\mathcal{I}$ is a mapping $f:[1, \ell] \times[1, n] \mapsto \Delta$ for some natural number $\ell>0$. Note that each row of a grid consists of $n$ cells and each cell contains a domino type. A tiling of $\mathcal{I}$ is a grid $f:[1, \ell] \times[1, n] \mapsto \Delta$ satisfying the following additional requirements. 
- two adjacent cells in a row have the same color on the shared edge, namely, for all $(i, j) \in[1, \ell] \times[1, n-1],[f(i, j)]_{\text {right }}=[f(i, j+1)]_{\text {left }}$ (row constraint);

- two adjacent cells in a column have the same color on the shared edge, namely, for all $(i, j) \in[1, \ell-1] \times[1, n],[f(i, j)]_{u p}=[f(i+1, j)]_{\text {down }}$ (column constraint);

- $f(1,1)=d_{\text {init }}($ initialization $)$ and $f(\ell, n)=d_{\text {final }}$ (acceptance).

Remark 5.3. Without loss of generality, we can assume that for all domino-types $d, d^{\prime} \in \Delta$ :

- if $[d]_{\text {right }}=\left[d^{\prime}\right]_{\text {left }}$, then $d \neq d_{\text {final }}$ and $d^{\prime} \neq d_{\text {init }}$;

- if $[d]_{\text {up }}=\left[d^{\prime}\right]_{\text {down }}$, then $d \neq d_{\text {final }}$ and $d^{\prime} \neq d_{\text {init }}$.

This ensures that the first cell of a tiling $f$ is the only one containing $d_{\text {init }}$, and the last cell of $f$ is the only one containing $d_{\text {final }}$.

It is well known that the problem of checking the existence of a tiling of $\mathcal{I}$ is PSPACEcomplete [Har92]. To prove the statement about MC of Theorem 5.1, we show how to construct, in polynomial time, a finite Kripke structure $\mathcal{K}_{\mathcal{I}}$ and a $\left.\mathrm{D}\right|_{\mathcal{H} \text { om }}$ formula $\Phi_{\mathcal{I}}$ such that there is a tiling of $\mathcal{I}$ if and only if $\mathcal{K}_{\mathcal{I}} \not \models \neg \Phi_{\mathcal{I}}$ (Subsection 5.1). As for the claim about satisfiability checking, we explain how to construct, in polynomial time for the given instance $\mathcal{I}$, a $\left.\mathrm{D}\right|_{\mathcal{H} \text { om }}$ formula which is satisfiable if and only if there is a tiling of $\mathcal{I}$ (Subsection 5.2).

First, we define a suitable encoding of the tilings of $\mathcal{I}$ by non-empty finite words over $2^{\mathscr{A} \mathcal{P}}$, where the set $\mathcal{A P}$ of atomic propositions is given by $\mathcal{A P}=\Delta \times[1, n] \times\{0,1\}$. In the following, we identify non-empty finite words $w$ over $2^{\not \mathcal{A P}}$ with the induced homogeneous interval models $\mathbf{M}(w)$.

We encode the row of a tiling by concatenating the codes of the row's cells starting from the first cell, and by marking the encoding with a tag which is a bit in $\{0,1\}$. Each cell code keeps track of the associated content and position along the row. Formally, a row-code with $\operatorname{tag} b \in\{0,1\}$ is a word over $2^{\mathfrak{A P}}$ of length $n$ having the form $\left\{\left(d_{1}, 1, b\right)\right\} \ldots\left\{\left(d_{n}, n, b\right)\right\}$ such that the following holds:

- for all $i \in[1, n-1],\left[d_{i}\right]_{\text {right }}=\left[d_{i+1}\right]_{\text {left }}$ (row constraint).

A sequence $\nu$ of row-codes is well-formed if for each non-last row-code in $\nu$ with tag $b$, the next row-code in $\nu$ has tag $1-b$ for all $b \in\{0,1\}$ (i.e., the tag changes in moving from a row-code to the next one). Tilings $f$ are then encoded by words over $2^{\mathcal{A P}}$ corresponding to well-formed concatenations of the codes of the rows of $f$ starting from the first row.

Note that by adding two additional padding colors $c_{L}$ and $c_{R}$ and domino types whose up and down parts are distinct from $c_{L}$ and $c_{R}$, we can also assume that each partial row-code $\left\{\left(d_{i}, i, b\right)\right\} \ldots\left\{\left(d_{j}, j, b\right)\right\}$, where $i \leq j, d_{i} \neq d_{\text {init }}$ if $i \neq 1$, and $d_{j} \neq d_{\text {final }}$ if $j \neq n$, can always be extended to a whole row-code.

5.1. PSPACE-hardness for $\mathbf{M C}$ against $\left.\mathbf{D}\right|_{\mathcal{H} \text { om }}$. Let as define an initialized well-formed sequence of row-codes as a sequence whose first symbol is $\left.\left\{\left(d_{\text {init }}, 1,0\right)\right\}\right)$. By construction, the following result trivially holds.

Proposition 5.4. A finite Kripke structure $\mathcal{K}_{\mathcal{I}}$ over $2^{\mathfrak{A P}}$ such that the initial traces $w$ of $\mathcal{K}$ correspond to the non-empty prefixes of the initialized well-formed sequences of row-codes can be built in time polynomial in the size of $\mathcal{I}$.

We now construct in polynomial time a $\left.\mathrm{D}\right|_{\mathcal{H} \text { om }}$ formula $\Phi_{\mathcal{I}}$ such that, given a non-empty prefix $w$ of an initialized well-formed sequence of row-codes, $w$ is a model of $\Phi_{\mathcal{I}}$ if and only if $w$ has some prefix encoding a tiling of $\mathcal{I}$. Hence, by Proposition 5.4, there exists a tiling 
of $\mathcal{I}$ if and only if there is some initial trace $w$ of $\mathcal{K}_{\mathcal{I}}$ such that $\mathcal{K}_{\mathcal{I}}, w=\Phi_{\mathcal{I}}$ if and only if $\mathcal{K}_{\mathcal{I}} \not \models \neg \Phi_{\mathcal{I}}$.

Let $w$ be a non-empty prefix of an initialized well-formed sequence of row-codes. Formula $\Phi_{\mathcal{I}}$ must enforce the acceptance requirement and the column constraint between adjacent row-codes. For acceptance, it suffices to force $w$ to visit some position where proposition $\left(d_{\text {final }}, n, b\right)$ holds for some tag $b \in\{0,1\}$. This can be done with the following $\left.\mathrm{D}\right|_{\mathcal{H} \text { om }}$ formula:

$$
\varphi_{\text {final }}:=\bigvee_{b \in\{0,1\}}\langle D\rangle\left(d_{\text {final }}, n, b\right)
$$

For the column constraint, it suffices to ensure the following requirement.

(C-Req): for each infix $\eta$ of $w$ of length $n+1$, let $\ell$ and $\ell^{\prime}$ be the unique two distinct positions of $\eta$, with $\ell$ preceding $\ell^{\prime}$, with the same cell index. Then, it holds that $\left[d_{\ell}\right]_{\text {up }}=\left[d_{\ell^{\prime}}\right]_{\text {down }}$, where $d_{\ell}$ (resp., $\left.d_{\ell^{\prime}}\right)$ is the domino-type of position $\ell$ (resp., $\left.\ell^{\prime}\right)$.

In order to express this requirement in $\left.\mathrm{D}\right|_{\mathcal{H} o m}$, we crucially observe that positions $\ell$ and $\ell^{\prime}$ of the infix $\eta$ of length $n+1$ have distinct tag. Moreover, $\ell$ precedes $\ell^{\prime}$ if and only if either (i) $\ell$ and $\ell^{\prime}$ have cell index $n$ and there is a position of $\eta$ with cell index 1 and the same tag as $\ell^{\prime}$, or (ii) $\ell$ and $\ell^{\prime}$ have cell index $i$, for some $i<n$, and there is a position of $\eta$ with cell index $i+1$ and the same tag as $\ell$. Requirement (C-Req) can thus be expressed by the following $\left.\mathrm{D}\right|_{\mathcal{H} \text { om }}$ formula:

$$
\begin{aligned}
\varphi_{C}:=[D]\left(\text { len }_{=n+1} \longrightarrow\right. & \bigvee_{d, d^{\prime} \in \Delta \mid[d]_{\text {up }}=\left[d^{\prime}\right]_{\text {down }}} \bigvee_{b \in\{0,1\}}\left[\left(\langle D\rangle(d, n, b) \wedge\langle D\rangle\left(d^{\prime}, n, 1-b\right) \wedge \bigvee_{i \in[1, n-1]}\left(\langle D\rangle(d, i, b) \wedge\langle D\rangle\left(d^{\prime}, i, 1-b\right) \wedge{\left.\left.d^{\prime \prime}, 1,1-b\right)\right) \vee}_{d^{\prime \prime} \in \Delta}\left(d^{\prime \prime}, i+1, b\right)\right)\right]\right)
\end{aligned}
$$

where $l e n_{=n+1}$ is the formula $\langle D\rangle^{n} \top \wedge[D]^{n+1} \perp$ capturing the infixes of length $n+1$. The desired formula $\Phi_{\mathcal{I}}$ is given by $\varphi_{\text {final }} \wedge \varphi_{C}$. This concludes the proof of the statement about MC of Theorem 5.1 (assuming the $\sqsubset$-semantics).

5.2. PSPACE-hardness for $\left.\mathbf{D}\right|_{\mathcal{H} \text { om }}$ satisfiability checking. For an instance $\mathcal{I}$ of the considered domino-tiling problem, we construct in polynomial time a $\left.\mathrm{D}\right|_{\mathcal{H} \text { om }}$ formula $\Psi_{\mathcal{I}}$ (under the $\sqsubset$-semantics) such that $\mathcal{I}$ has some tiling if and only if $\Psi_{\mathcal{I}}$ is satisfiable.

To build the formula $\Psi_{\mathcal{I}}$, we exploit as conjuncts the two $\left.\mathrm{D}\right|_{\mathcal{H} \text { om }}$ formulas $\varphi_{\text {final }}$ and $\varphi_{C}$, ensuring the acceptance requirement and the column constraint, respectively, given in Subsection 5.1. Additionally, we introduce as a third conjunct in the definition of $\Psi_{\mathcal{I}}$, the $\left.\mathrm{D}\right|_{\mathcal{H} o m}$ formula $\varphi_{w f}$, which, intuitively, "emulates" the behaviour of the Kripke structure $\mathcal{K}_{\mathcal{I}}$ in Proposition 5.4. Thus, $\Psi_{\mathcal{I}}:=\varphi_{w f} \wedge \varphi_{\text {final }} \wedge \varphi_{C}$.

The models of $\varphi_{w f}$ are all and only the non-empty finite words $w$ over $2^{\mathscr{A P}}$ satisfying the following condition: either $w$ or its reverse $w^{R}$ coincides with the infix $\eta$ of some well-formed sequence of row-codes such that (i) $\eta$ visits some cell code with cell index 1 and content $d_{\text {init }}$, and (ii) $\eta$ contains some row-code. By construction (see Subsection 5.1), in both cases $(w=\eta$ or $w^{R}=\eta$ ) the conjunct $\varphi_{C}$ in the definition of $\Psi_{\mathcal{I}}$ enforces the column constraint on $\eta$. Moreover, our assumption on the instance $\mathcal{I}$ (see Remark 5.3) entails that the $d_{\text {final }}$-positions in $\eta$ follow the $d_{\text {init }}$-positions. This ensures that (i) for each model $w$ of $\psi_{\mathcal{I}}$, either $w$ or its reverse $w^{R}$ has an infix encoding a tiling of $\mathcal{I}$, and (ii) each tiling of $\mathcal{I}$ is a model of $\Psi_{\mathcal{I}}$. 
We now provide the technical details of the construction of $\varphi_{w f}$, which is the conjunction of the following subformulas (w.l.o.g., we assume $n>2$ ):

(1) Propositional mutual exclusion: at every position, at most one proposition letter holds. This is expressed by the $\left.\mathrm{D}\right|_{\mathcal{H} \text { om }}$ formula

$$
\neg \bigvee_{p, p^{\prime} \in \mathcal{A} \mathscr{P} \mid p \neq p^{\prime}}\langle D\rangle\left(p \wedge p^{\prime}\right)
$$

(2) Initialization: there is some position where $\left(d_{\text {init }}, 1, b\right)$ holds for some tag $b \in\{0,1\}$. This is captured by the in $\left.\mathrm{D}\right|_{\mathcal{H} o m}$ formula

$$
\bigvee_{b \in\{0,1\}}\langle D\rangle\left(d_{\text {init }}, 1, b\right)
$$

(3) $n$-length constraint: there is some infix of the given word $w$ ( $w$ included) having length $n$ and such that all its positions have the same tag. This requirement can be formalized in $\left.\mathrm{D}\right|_{\mathcal{H} o m}$ as $\langle D\rangle \psi \vee \psi$, where $\psi$ is the $\left.\mathrm{D}\right|_{\mathcal{H} o m}$ formula

$$
\psi:=\operatorname{len}_{=n} \wedge \bigvee_{b \in\{0,1\}}[D]\left(\operatorname{len}_{=1} \rightarrow \bigvee_{d \in \Delta} \bigvee_{i \in[1, n]}(d, i, b)\right)
$$

(4) Fixed ordering of cell indexes: for each infix $\eta$ of the given word $w$ ( $w$ included) having length $n$ and for each cell index $i \in[1, n]$, there is some position of $\eta$ with cell index $i$. This requirement ensures that there is a bijection (permutation) $\pi$ over $[1, n]$ such that the projection of $w$ over the $[1, n]$-component of $\mathscr{A P}$ has the form

$$
\pi(i+1) \ldots \pi(n)[\pi(1) \ldots \pi(n)]^{*} \pi(1) \ldots \pi(j-1) \text { for some } i, j \in[1, n]
$$

Such a requirement can be expressed as $[D] \xi \wedge \xi$ where $\xi$ is the $\left.\mathrm{D}\right|_{\mathcal{H} \text { om }}$ formula

$$
\xi:=l e n_{=n} \longrightarrow \bigwedge_{i \in[1, n]}\langle D\rangle \bigvee_{d \in \Delta} \bigvee_{b \in\{0,1\}}(d, i, b)
$$

Note that Requirements (1) and (4) also ensure that exactly one proposition letter holds at each position.

(5) Correct ordering of cell indexes, row constraint, and well-formedness: we require that for each infix $\eta$ of the given word $w$ having length 2, the following holds:

- either the two positions of $\eta$ have the same tag and consecutive cell indexes $i$ and $i+1$ for some $1 \leq i<n$ such that $\left[d_{i}\right]_{\text {right }}=\left[d_{i+1}\right]_{\text {left }}$, where $d_{i}$ (resp., $d_{i+1}$ ) is the domino-type of position with cell index $i$ (resp., $i+1$ ),

- or the two positions of $\eta$ have distinct tag and cell indexes 1 and $n$, respectively.

This ensures, in particular, that the permutation $\pi$ in Requirement (4) corresponds either to the natural ordering of cell indexes or to its reverse. Requirement (4) can be easily captured by the $\left.\mathrm{D}\right|_{\mathcal{H} \text { om }}$ formula

$$
\begin{aligned}
\varphi_{C}:= & {[D](\text { len }=2 \longrightarrow} \\
& {\left[\bigvee_{d, d^{\prime} \in \Delta \mid[d]_{\text {right }}=\left[d^{\prime}\right]_{l e f t}} \bigvee_{b \in\{0,1\}} \bigvee_{i \in[1, n-1]}\left(\langle D\rangle(d, i, b) \wedge\langle D\rangle\left(d^{\prime}, i+1, b\right)\right) \vee\right.} \\
& \left.\bigvee_{d, d^{\prime} \in \Delta} \bigvee_{b \in\{0,1\}}\left(\langle D\rangle(d, 1, b) \wedge\langle D\rangle\left(d^{\prime}, n, 1-b\right)\right)\right]
\end{aligned}
$$


By construction, it easily follows that a word $w$ is a model of $\varphi_{w f}$ if and only if (i) $w$ visits some position with the initial domino-type and cell index 1, and (ii) $w$ or its reverse corresponds to an infix $\eta$ of some well-formed sequence of row-codes such that $\eta$ contains some row-code. This concludes the proof of the statement about satisfiability checking of Theorem 5.1 (assuming the $\sqsubset$-semantics).

\section{Conclusions}

In this paper, we have determined the exact complexity of the satisfiability checking and model checking problem for the logic D of sub-intervals over finite linear orders and finite Kripke structures, respectively, under the homogeneity assumption. Since the two problems have been proved to be PSPACE-complete, we have enriched the set of known "tractable" fragments of HS (see $\left[\mathrm{BMM}^{+} 19 \mathrm{~b}\right]$ ) with a meaningful logic.

The tractability of D gives an additional insight in the open problem of the existence of elementary decision procedures for model checking the logic BE and the full logic HS. D can be considered as the most meaningful logic included in BE, and its tractability leaves open the possibility of a positive answer to the open question.

A way to tackle the question is to investigate the HS fragments featuring the modality $\mathrm{D}$ together with either the modality B (for the relation started-by) or the modality $\mathrm{E}$ (for the relation finished-by) so to gradually approach the expressive power of BE.

The complexity of model checking the logic BE and the full HS logic remains the most intriguing and challenging open question.

Acknowledgements. The work by Alberto Molinari, Angelo Montanari, and Pietro Sala has been supported by the GNCS project Logic and Automata for Interval Model Checking.

\section{REFERENCES}

$\left[\mathrm{BDG}^{+} 14\right]$ D. Bresolin, D. Della Monica, V. Goranko, A. Montanari, and G. Sciavicco. The dark side of interval temporal logic: marking the undecidability border. Annals of Mathematics and Artificial Intelligence, 71(1-3):41-83, 2014. doi:10.1007/s10472-013-9376-4.

[BGMS09] D. Bresolin, V. Goranko, A. Montanari, and G. Sciavicco. Propositional interval neighborhood logics: Expressiveness, decidability, and undecidable extensions. Annals of Pure and Applied Logic, 161(3):289-304, 2009. doi:10.1016/j.apal.2009.07.003.

[BGMS10] D. Bresolin, V. Goranko, A. Montanari, and P. Sala. Tableaux for logics of subinterval structures over dense orderings. Journal of Logic and Computation, 20(1):133-166, 2010. doi:10.1093/ logcom/exn063.

$\left[\mathrm{BMM}^{+} 17\right] \quad$ L. Bozzelli, A. Molinari, A. Montanari, A. Peron, and P. Sala. Satisfiability and Model Checking for the Logic of Sub-Intervals under the Homogeneity Assumption. In Proceedings of the 44th International Colloquium on Automata, Languages, and Programming (ICALP), pages 120:1120:14. Schloss Dagstuhl-Leibniz-Zentrum fuer Informatik, 2017. doi:10.4230/LIPIcs. ICALP. 2017.120.

$\left[\mathrm{BMM}^{+} 18\right] \quad$ L. Bozzelli, A. Molinari, A. Montanari, A. Peron, and P. Sala. Model checking for fragments of the interval temporal logic HS at the low levels of the polynomial time hierarchy. Information and Compututation, 262(Part):241-264, 2018. doi:10.1016/j.ic.2018.09.006.

$\left[\mathrm{BMM}^{+} 19 \mathrm{a}\right]$ L. Bozzelli, A. Molinari, A. Montanari, A. Peron, and P. Sala. Interval vs. point temporal logic model checking: An expressiveness comparison. ACM Trans. Comput. Log., 20(1):4:1-4:31, 2019. doi:10.1145/3281028.

$\left[\mathrm{BMM}^{+} 19 \mathrm{~b}\right]$ L. Bozzelli, A. Molinari, A. Montanari, A. Peron, and P. Sala. Which fragments of the interval temporal logic HS are tractable in model checking? Theoretical Computer Science, 764:125-144, 2019. doi:10.1016/j.tcs.2018.04.011. 
[DGMS11] D. Della Monica, V. Goranko, A. Montanari, and G. Sciavicco. Interval temporal logics: a journey. Bulletin of the EATCS, 105:73-99, 2011. URL: http://albcom.1si.upc.edu/ojs/ index.php/beatcs/article/view/98.

[Har92] D. Harel. Algorithmics: The spirit of computing. Wesley, 2nd edition, 1992.

[HS91] J. Y. Halpern and Y. Shoham. A propositional modal logic of time intervals. Journal of the ACM, 38:279-292, 1991. doi:10.1145/115234.115351.

[KR93] H. Kamp and U. Reyle. From Discourse to Logic: Introduction to Model-theoretic Semantics of Natural Language, Formal Logic and Discourse Representation Theory, Volume 42 of Studies in Linguistics and Philosophy. Springer, 1993. doi:10.1007/978-94-017-1616-1.

[LM13] A. Lomuscio and J. Michaliszyn. An epistemic Halpern-Shoham logic. In Proceedings of the 23rd International Joint Conference on Artificial Intelligence (IJCAI), pages 1010-1016, 2013.

[LM14] A. Lomuscio and J. Michaliszyn. Decidability of model checking multi-agent systems against a class of EHS specifications. In Proceedings of the 21st European Conference on Artificial Intelligence (ECAI), pages 543-548, 2014. doi:10.3233/978-1-61499-419-0-543.

[LM16] A. Lomuscio and J. Michaliszyn. Model checking multi-agent systems against epistemic HS specifications with regular expressions. In Proceedings of the 15th International Conference on Principles of Knowledge Representation and Reasoning (KR), pages 298-308, 2016.

[Lod00] K. Lodaya. Sharpening the undecidability of interval temporal logic. In Proceedings of the 6th Asian Computing Science Conference (ASIAN), pages 290-298, 2000. doi:10.1007/ 3-540-44464-5_21.

[MM14] J. Marcinkowski and J. Michaliszyn. The undecidability of the logic of subintervals. Fundamenta Informaticae, 131(2):217-240, 2014. doi:10.3233/FI-2014-1011.

$\left[\mathrm{MMM}^{+}{ }^{16}\right]$ A. Molinari, A. Montanari, A. Murano, G. Perelli, and A. Peron. Checking interval properties of computations. Acta Informatica, 53(6-8):587-619, 2016. doi:10.1007/s00236-015-0250-1.

[MMP18] A. Molinari, A. Montanari, and A. Peron. Model checking for fragments of halpern and shoham's interval temporal logic based on track representatives. Information and Computation, 259(3):412-443, 2018. doi:10.1016/j.ic.2017.08.011.

[Mon16] A. Montanari. Interval temporal logics model checking. In Proceedings of the 23rd International Symposium on Temporal Representation and Reasoning, (TIME), page 2, 2016. doi:10.1109/ TIME. 2016.32.

[MPS10] A. Montanari, I. Pratt-Hartmann, and P. Sala. Decidability of the logics of the reflexive sub-interval and super-interval relations over finite linear orders. In Proceedings of the 17th International Symposium on Temporal Representation and Reasoning (TIME), pages 27-34, 2010. doi:10.1109/TIME.2010.18.

[Ott01] M. Otto. Two variable first-order logic over ordered domains. Journal of Symbolic Logic, 66(2):685-702, 2001. doi:10.2307/2695037.

[Pra05] Ian Pratt-Hartmann. Temporal prepositions and their logic. Artificial Intelligence, 166(1-2):1-36, 2005. doi:10.1016/j.artint.2005.04.003.

[Sha04] I. Shapirovsky. On PSPACE-decidability in transitive modal logic. In Proceedings of the 5th conference on Advances in Modal logic (AiML), pages 269-287, 2004. URL: http://www. aiml. net/volumes/volume5/Shapirovsky.ps.

[SS05] I. Shapirovsky and V. B. Shehtman. Modal Logics of Regions and Minkowski Spacetime. Journal of Logic and Computation, 15(4):559-574, 2005. doi:10.1093/logcom/exi039.

[vB91] J. van Benthem. Language in action. Journal of Philosophical Logic, 20(3):225-263, 1991. doi: 10.1007/BF00250539.

[Ven90] Y. Venema. Expressiveness and completeness of an interval tense logic. Notre Dame Journal of Formal Logic, 31(4):529-547, 1990. doi:10.1305/ndjfl/1093635589.

[Ven91] Y. Venema. A modal logic for chopping intervals. Journal of Logic and Computation, 1(4):453476, 1991. doi:10.1093/logcom/1.4.453. 